\title{
A new approach to calculating the memory kernel of the generalized quantum master equation for an arbitrary system-bath coupling
}

\author{
Qiang Shi and Eitan Geva ${ }^{a}$ \\ Department of Chemistry and the FOCUS Center, University of Michigan, Ann Arbor, Michigan 48109-1055
}

(Received 17 July 2003; accepted 19 September 2003)

\begin{abstract}
The Nakajima-Zwanzig generalized quantum master equation provides a general, and formally exact, prescription for simulating the reduced dynamics of a quantum system coupled to a quantum bath. In this equation, the memory kernel accounts for the influence of the bath on the system's dynamics. The standard approach is based on using a perturbative treatment of the system-bath coupling for calculating this kernel, and is therefore restricted to systems weakly coupled to the bath. In this paper, we propose a new approach for calculating the memory kernel for an arbitrary system-bath coupling. The memory kernel is obtained by solving a set of two coupled integral equations that relate it to a new type of two-time system-dependent bath correlation functions. The feasibility of the method is demonstrated in the case of an asymetrical two-level system linearly coupled to a harmonic bath. (C) 2003 American Institute of Physics. [DOI: 10.1063/1.1624830]
\end{abstract}

\section{INTRODUCTION}

Quantum dynamical effects play a central role in a variety of important processes that take place in condensed phase environments. ${ }^{1-3}$ Important examples, which are particularly relevant to chemistry, include proton and electron transfer reactions,${ }^{4-17}$ intramolecular vibrational relaxation, ${ }^{18-43}$ and optical and infrared chromophore spectroscopy and photochemistry. ${ }^{44-79}$

The simulation of quantum dynamics in condensed phase hosts is one of the most important challenges facing theoretical chemistry. A numerically exact solution of the Schrödinger equation ${ }^{80-84}$ in the case of general many-body systems remains far beyond the reach of currently available computer resources, due to the exponential scaling of the computational effort with the number of degrees of freedom (DOF). A common approach for dealing with this difficulty focuses on computing more reduced quantities, which are directly related to the relevant experimental probes. Two such, inter related, reductionist strategies have been found to be particularly useful in practice:

(i) Description in terms of the reduced density operator $(\mathrm{RDO})^{85}$ of a relatively low dimensional subsystem, which is subject to direct experimental manipulation and/or observation. The subsystem may correspond to the reaction coordinate in an electron or proton transfer reaction, a relaxing vibrational mode of a solute molecule, or an optically active transition in a solvated chromophore molecule. One may then employ a strategy which combines an accurate description of the subsystem's quantum dynamics, with a minimal treatment of the dynamics of the rest of the DOF (the bath), to the extent that will make it possible to accurately capture their effect on the subsystem of interest.

(ii) Description in terms of time correlation functions (TCFs), which represent much simpler, and averaged over,

\footnotetext{
a) Author to whom correspondence should be addressed. Electronic mail: eitan@umich.edu
}

mathematical constructs in comparison to multidimensional wave functions. Thus, a reaction rate constant may be written in terms of the flux-flux correlation function, ${ }^{4,5}$ a vibrational energy relaxation rate constant may be put in terms of the force-force correlation function, ${ }^{18-20}$ and spectroscopic response functions are often expressed in terms of dipole correlation functions. ${ }^{57}$ Those time correlation functions can obviously be obtained by averaging over the multidimensional wave functions of the overall system. However, the true challenge lies in finding effective, computationally feasible, and versatile methods for calculating quantum-mechanical TCFs, by taking advantage of their more averaged nature, in order to avoid the obvious, yet hopeless, wave-function-based route.

The starting point of the RDO approach is based on finding compact, yet effective, ways for characterizing the influence of the bath on the subsystem's dynamics. One strategy is based on finding the equation of motion that governs the dynamics of the RDO. This equation must include a dissipative term that accounts for the influence of the bath. Several versions of the exact reduced equation of motion, also known as the generalized quantum master equation (GQME), have been proposed ${ }^{86-94}$ However, the use of those GQMEs as such, has been scarce due to the complexity of the dissipative term. As a result, those GQMEs have been mostly used as the starting point of more approximate treatments, which lead to a more manageable dissipative term. A very popular approach is based on the assumption that the subsystem is weakly coupled to the bath, and that its relaxation occurs on a time scale which is much longer than that of the bath fluctuations. This results in a Markovian quantum master equation (QME), where the influence of the bath shows up via population and phase relaxation rate constants (note that the name QME is reserved in this paper for the weak coupling limit of the GQME) ${ }^{85,91,93,95-115}$ In fact, the latter can be expressed in terms of two-time free-bath TCFs, and as such form a bridge between the RDO and TCF ap- 
proaches. However, one must not lose sight of the fact that the assumptions of weak coupling and Markovity impose serious restrictions on the range of phenomena that can be captured by QMEs. For example, QMEs, either Markovian or non-Markovian, are unable to describe such important phenomena as solvation dynamics and solvent memory effects, which are central to solution chemistry.

It should also be noted that the derivation of the QME has been extended so as to account for fourth order terms in the system-bath coupling ${ }^{104,116-119}$ (as opposed to the standard derivation, which only accounts for second order terms). Most applications of this approach were restricted to the spin-boson problem, where important insight has been gained. However, the fact that the fourth order terms are generally expressed in terms of triple integrals over four-time free-bath TCFs, would make a general computational implementation of this scheme rather difficult. An alternative approach has been based on optimizing the separation of the overall system into system and bath, such that weak coupling is satisfied. ${ }^{106}$ This approach is most effective when one can identify a few local or collective bath coordinates which are strongly coupled to the system of interest, while weakly coupled to the other bath modes, and include them in the system Hamiltonian. ${ }^{120,121}$ Alternatively, one may attempt a canonical transformation of the overall Hamiltonian, that may lead to newly defined, and presumably weakly coupled, system and bath terms, which mix the original system and bath coordinates. ${ }^{122}$ Both approaches have been demonstrated with impressive success in the spin-boson case, where it is relatively easy to identify the above mentioned strongly coupled bath coordinate, or come up with the suitable canonical transformation. Unfortunately, accomplishing this for more complex and anharmonic systems appears to be far more difficult, and, to the best of our knowledge, represents an unresolved problem at the present time. Another recent attempt at extending the applicability of QMEs beyond the weak coupling limit, has been based on an approximate resummation of all the terms in the perturbation expansion, to infinite order. ${ }^{123-125}$ Although this approach was found to yield accurate predictions in the context of charge transfer, the general applicability of the approximations underlying it is not clear at the present time.

An alternative to the GQME-based approach, which gained popularity over the last several decades, is based on the path integral formulation of quantum mechanics, ${ }^{126-128}$ and introduces the influence of the bath in terms of an influence functional (IF). ${ }^{129}$ One of the most impressive triumphs of this approach over the QME approach, had to do with the fact that the exact IF can be obtained in closed form, in the case of linear coupling to a harmonic bath. ${ }^{130-133}$ This fact, in conjunction with important algorithmic advances, such as the development of iterative tensor quasiadiabatic propagators (QUAPIs) by Makri and co-workers, have opened the door to numerically exact calculations of the reduced dynamics of this type of systems (as long as one can evaluate the remaining path integral over the subsystem DOF). ${ }^{10,134-148}$ However, there are many important systems, e.g., liquid solutions, where it is difficult to map the bath Hamiltonian onto a harmonic one. Recent attempts by Makri and co-workers to use semiclassical approximations in order to evaluate the IF in the case of anharmonic baths and nonlinear coupling ${ }^{11,149,150}$ appear promising, although their general applicability remains unclear at this preliminary stage of their development.

The TCF approach relies on finding a relationship between nonequilibrium quantum dynamics and TCFs, which characterize the equilibrium dynamics of the system. One relationship, which has been pointed out above, put the population and phase relaxation rate coefficients of a subsystem weakly coupled to a bath in terms of free-bath TCFs. For example, the expression for the vibrational energy relaxation rate constant in terms of a force-force correlation function, falls into this category. Another, distinctly different type of relationship can be established with the help of linearresponse theory (LRT). The latter is based on the fact that the nonequilibrium relaxation dynamics of a system in the close vicinity of equilibrium, is the same as that of the relaxation of its spontaneous fluctuations around equilibrium, i.e., of the corresponding TCF. ${ }^{103}$ It is important to note that LRT does not require a separation of the overall system into a subsystem and a bath, and obviously does not make any assumption regarding their coupling. However, LRT is restricted to systems in the close vicinity of thermal equilibrium, which implies that it will be particularly useful in two situations: (1) when the system is subject to a relatively weak external perturbation, which shifts the system only slightly relative to its equilibrium state and (2) when the system follows rate kinetics, such that the rate constant does not depend on the initial state, and can therefore be conveniently calculated with an initial state which is in the close vicinity of equilibrium. An important example for the first scenario is provided by linear spectroscopy, where the laser field is often assumed to be a small perturbation. For example, the absorption spectrum can be expressed in terms of a two-time dipole TCF. In fact, this approach can be extended so as to account for higher order nonlinear response to the laser field, and put it in terms of multitime dipole TCFs. ${ }^{57}$ An important example of the second scenario is given by chemical reactions, where rare event statistics associated with barrier crossing leads to rate kinetics. ${ }^{4,15}$

The development of methods for computing quantummechanical TCFs continues to be at the forefront of theoretical and computational physical chemistry research. Several strategies have been proposed in order to address the challenge of providing an effective, computationally feasible, and versatile approximate method for calculating quantummechanical TCFs. Those methods are based on various approaches, including a mixed quantum-classical treatment, ${ }^{151-156}$ analytical continuation, ${ }^{12-14,38,157-162}$ centroid molecular dynamics, $9,15,16,37,42,163-175$ quantum mode coupling theory, ${ }^{161,176-179}$ and the semiclassical (SC) approximation. ${ }^{2,11,180-197}$

The various approaches mentioned above provide powerful tools for dealing with a wide variety of quantum phenomena that occur in condensed-phase systems. However, the assumptions upon which they are based, also make it difficult to apply those methods to many other important problems. For example, the computation of the multitime 
dipole correlation functions, required for analyzing nonlinear optical experiments in liquid solutions, can be very costly, even after the implementation of rather severe approximations. It is also becoming increasingly important to consider the impact of intense laser fields, e.g., in the area of coherent control. ${ }^{198-202}$ In addition to that, many relaxation processes cannot be characterized by rate constants. A direct treatment in terms of the nonequilibrium dynamics of the RDO appears to offer an attractive alternative in such situations. However, QMEs are restricted to systems weakly coupled to their environments, and miss many important phenomena as a result.

The dilemma described above provided the motivation for the work reported in the present paper. Below, we propose a new approach which goes back to the formally exact GQME, and addresses the question of computing its dissipative part without resorting to the assumptions of weak system-bath coupling and Markovity. The analysis is performed in terms of the Nakajima-Zwanzig formulation of the GQME, ${ }^{86-94,97-101}$ where the problem of evaluating the dissipative part reduces to computing a memory kernel superoperator. The strategy we propose is based on expressing this kernel in terms of two-time system-dependent TCFs, which should be contrasted to the free-bath TCFs that the perturbative approach gives rise to. As such, the new approach provides a completely general relationship between the RDO and TCF approaches, which goes beyond currently available LRT-based and QME-based theories, and which is applicable to systems that are not accessible to them.

The structure of the remainder of this paper is as follows. The theoretical framework underlying the NakajimaZwanzig GQME is outlined in Sec. II. A new formulation of its memory kernel in terms of system-dependent TCFs is presented in Sec. III. The feasibility of the method is demonstrated in the case of a system linearly coupled to a harmonic bath in Sec. IV. The main conclusions are summarized, and their significance is discussed in Sec. V. Useful identities and proofs for some of the formal results used in the text are provided in Appendices A, B, and C.

\section{PRELIMINARY CONSIDERATIONS}

We consider a system with the following general quantum-mechanical Hamiltonian:

$$
\hat{H}=\hat{H}_{s}+\hat{H}_{b}+\hat{H}_{\mathrm{bs}},
$$

where $\hat{H}_{s}$ is the Hamiltonian of the subsystem of interest, which will be referred to as the system from here on, $\hat{H}_{b}$ is the Hamiltonian of the remaining DOF, which will be referred to as the bath from here on, and $\hat{H}_{\mathrm{bs}}$ is the coupling between the system and the bath. For simplicity, we assume the following form for the latter:

$$
\hat{H}_{\mathrm{bs}}=\hat{\Lambda} \otimes \hat{F},
$$

where $\hat{\Lambda}$ is a bath operator and $\hat{F}$ is a system operator (a generalization of the results to the case $\hat{H}_{\mathrm{bs}}=\Sigma_{j} \hat{\Lambda}_{j} \otimes \hat{F}_{j}$ is particularly straightforward when $\left[\hat{F}_{j}, \hat{F}_{k}\right]=0$ for all $j$ and $k$, as in the common case where $\left\{\hat{F}_{j}\right\}$ are all functions of the system coordinates). The states of the overall system and subsystem at time $t$ are completely defined by the density operators $\hat{\rho}(t)$ and

$$
\hat{\rho}_{s}(t)=\operatorname{Tr}_{b} \hat{\rho}(t),
$$

respectively, where $\operatorname{Tr}_{b}$ corresponds to a partial trace over the Hilbert space of the bath.

The initial state of the overall system is assumed factorized,

$$
\hat{\rho}(0)=\hat{\rho}_{s}(0) \otimes \hat{\rho}_{b}^{\mathrm{eq}},
$$

where $\hat{\rho}_{s}(0)$ is the initial density operator of the system, and

$$
\hat{\rho}_{b}^{\mathrm{eq}}=e^{-\beta \hat{H}_{b}} / \operatorname{Tr}_{b}\left[e^{-\beta \hat{H}_{b}}\right]
$$

is the density operator of the free bath at thermal equilibrium. It should be noted that the choice of a factorized initial state, Eq. (4), does not limit the generality of the treatment, since it is always possible to alter the initial state via preliminary equilibration and/or the use of an additional driving term in the system Hamiltonian. Finally, it is assumed that

$$
\langle\hat{\Lambda}\rangle_{\mathrm{eq}}^{0} \equiv \operatorname{Tr}_{b}\left[\hat{\rho}_{b}^{\mathrm{eq}} \hat{\Lambda}\right]=0
$$

[if originally $\langle\hat{\Lambda}\rangle_{\mathrm{eq}}^{0} \neq 0$, one can still satisfy this condition by substituting $\hat{\Lambda}-\langle\hat{\Lambda}\rangle_{\mathrm{eq}}^{0}$ for $\hat{\Lambda}$, and $\hat{H}_{s}+\langle\hat{\Lambda}\rangle_{\mathrm{eq}}^{0} \hat{F}$ for $\hat{H}_{s}$ in Eq. (1)].

As is well known, the reduced quantum dynamics of the system can be described by the formally exact NakajimaZwanzig GQME, ${ }^{86-91,93,94}$ which assumes the following form under the conditions described above (in the Schrödinger picture):

$$
\frac{d}{d t} \hat{\rho}_{s}(t)=-\frac{i}{\hbar} \mathcal{L}_{s} \hat{\rho}_{s}(t)-\int_{0}^{t} d \tau \mathcal{K}(\tau) \hat{\rho}_{s}(t-\tau) .
$$

Here, $\mathcal{L}_{s}(\cdot)=\left[\hat{H}_{s}, \cdot\right]$ and $\int_{0}^{t} d \tau \mathcal{K}(\tau) \hat{\rho}_{s}(t-\tau)$ represent the bath-free (Hamiltonian) and bath-induced (non-Hamiltonian) contributions to the system dynamics, respectively. The memory kernel, $\mathcal{K}(\tau)$, is explicitly given by

$$
\mathcal{K}(\tau)=\frac{1}{\hbar^{2}} \operatorname{Tr}_{b}\left\{\mathcal{L}_{\mathrm{bs}} e^{-i \mathcal{Q} \mathcal{L} \tau / \hbar} \mathcal{Q} \mathcal{L}_{\mathrm{bs}} \hat{\rho}_{b}^{\mathrm{eq}}\right\},
$$

where $\mathcal{L}(\cdot)=[\hat{H}, \cdot], \mathcal{L}_{\mathrm{bs}}(\cdot)=\left[\hat{H}_{\mathrm{bs}}, \cdot\right], \mathcal{Q}=1-\mathcal{P}$, and

$$
\mathcal{P}(\cdot)=\hat{\rho}_{b}^{\mathrm{eq}} \otimes \operatorname{Tr}_{b}(\cdot) .
$$

It should be noted that $\mathcal{Q}$ and $\mathcal{P}$ are complementary projection superoperators $\left(\mathcal{Q}^{2}=\mathcal{Q}, \mathcal{P}^{2}=\mathcal{P}\right.$, and $\left.\mathcal{P} \mathcal{Q}=\mathcal{Q P}=0\right)$, and that $\mathcal{P}$ is defined with $\hat{\rho}_{b}^{\text {eq }}$ as the reference state. ${ }^{91} \mathrm{We}$ also note that the inhomogeneous term is missing from the GQME in Eq. (7) because of our choice of factorized, and hence uncorrelated, initial state. ${ }^{91}$

The main advantage of the GQME, Eq. (7), has to do with the fact that solving it would yield the exact quantum dynamics of the system. The dynamics of the system is no longer dictated solely by the bath-free system Hamiltonian, $\hat{H}_{s}$, and requires the Nakajima-Zwanzig memory kernel $\mathcal{K}(\tau)$, Eq. (8), as additional input. It should be noted that in many cases, one is not interested in a detailed description of the bath dynamics as such, but rather in its influence on the 
system dynamics. Thus, the memory kernel essentially filters out those aspects of the bath dynamics which influence the system, and which can actually be probed by performing measurements on the system. As such, it may be viewed as analogous to the IF in the path-integral approach. However, it is interesting to note that the time dependence of $\mathcal{K}(\tau)$ is simpler, being a function of a single time variable, rather than a functional of the system's path.

Unfortunately, the exact memory kernel is difficult to compute in practice since it involves dynamics which are induced by the projected Liouvillian of the overall system, $\mathcal{Q L}$. The Nakajima-Zwanzig GQME has therefore been used mostly as the starting point for more approximate treatments, which lead to simplified versions of the memory kernel. The most popular approach involves the assumption of weak system-bath coupling, which leads to the following approximation:

$$
\mathcal{K}(\tau) \approx \mathcal{K}_{\mathrm{wC}}(\tau)=\frac{1}{\hbar^{2}} \operatorname{Tr}_{b}\left\{\mathcal{L}_{\mathrm{bs}} e^{-i \mathcal{L}_{s} \tau / \hbar} e^{-i \mathcal{L}_{b} \tau / \hbar} \mathcal{L}_{\mathrm{bs}} \hat{\rho}_{b}^{\mathrm{eq}}\right\} .
$$

This leads to a dissipative part of the following form:

$$
\begin{aligned}
\int_{0}^{t} d \tau \mathcal{K}(\tau) \hat{\rho}_{s}(t-\tau) \\
\approx \int_{0}^{t} d \tau \mathcal{K}_{\mathrm{WC}}(\tau) \hat{\rho}_{s}(t-\tau) \\
=\int_{0}^{t} d \tau\left\{C(\tau)\left[\hat{F},\left(e^{-i \mathcal{L}_{s} \tau / \hbar} \hat{F} \hat{\rho}_{s}(t-\tau)\right)\right]+C(-\tau)\right. \\
\left.\quad \times\left[\left(e^{-i \mathcal{L}_{s} \tau / \hbar} \hat{\rho}_{s}(t-\tau) \hat{F}\right), \hat{F}\right]\right\},
\end{aligned}
$$

where

$$
C(\tau)=\left\langle e^{i \hat{H}_{b} t / \hbar} \hat{\Lambda} e^{-i \hat{H}_{b} t / \hbar} \hat{\Lambda}\right\rangle_{\mathrm{eq}}^{0}
$$

is a free-bath correlation function (FBCF).

The weak coupling approximation is often accompanied by the complementary assumption that the dissipative system dynamics is much slower than the bath correlation time, $\tau_{c}$, which is defined as the characteristic decay time of $C(\tau)$ (note that $\lim _{\tau \rightarrow \infty} C(\tau)=0$, since $\langle\hat{\Lambda}\rangle_{\mathrm{eq}}^{0}=0$ ). Under those conditions, one may extend the upper limit of the time integral to $\infty$ (for $t>\tau_{c}$ ), and substitute $e^{i \mathcal{L}_{s} \tau / \hbar} \hat{\rho}_{s}(t)$ for $\hat{\rho}_{s}(t$ $-\tau)$ in Eq. (11). This results in a Markovian QME of the following form:

$$
\frac{d}{d t} \hat{\rho}_{s}(t)=-\frac{i}{\hbar} \mathcal{L}_{s} \hat{\rho}_{s}(t)-\mathcal{L}_{D} \hat{\rho}_{s}(t),
$$

where

$$
\begin{aligned}
\mathcal{L}_{D} \hat{\rho}_{s}(t)= & \int_{0}^{\infty} d \tau\left\{C(\tau)\left[\hat{F},\left(e^{-i \mathcal{L}_{s} \tau / \hbar} \hat{F}\right) \hat{\rho}_{s}(t)\right]+C(-\tau)\right. \\
& \left.\times\left[\hat{\rho}_{s}(t)\left(e^{-i \mathcal{L}_{s} \tau / \hbar} \hat{F}\right), \hat{F}\right]\right\} .
\end{aligned}
$$

$\mathcal{L}_{D}$ can be put in the Bloch-Redfield form, ${ }^{95,96}$ by expressing $\hat{F}$ in terms of the eigenprojectors of $\hat{H}_{s}$

$$
\hat{F}=\sum_{k} F_{k l}|k\rangle\langle l|
$$

where $\hat{H}_{s}|k\rangle=\epsilon_{k}|k\rangle$. The effect of the bath may then be expressed in terms of quantities of the form $\int_{0}^{\infty} d \tau e^{i \omega_{k l} \tau} C(\tau)$, where $\omega_{k l}=\left(\epsilon_{k}-\epsilon_{l}\right) / \hbar$. More specifically, population and phase relaxation rate constants can be expressed in terms of the real parts of those integrals $\left(\omega_{k l}=0\right.$ and $\omega_{k l} \neq 0$ correspond to phase and population relaxation rate constants, respectively), while Lamb-type shifts that modify the Hamiltonian dynamics are associated with the imaginary parts.

It is important to note that the $\mathrm{FBCF}, C(\tau)$, embodies all the information needed for determining the influence of the bath on the system, provided that the two are weakly coupled. This implies that the nonequilibrium dynamics of the system can be inferred from the free bath equilibrium dynamics, which $C(\tau)$ is a measure of. This represents a great simplification, since the correlation time is often found to be much shorter than the system relaxation lifetimes. Indeed, it is rather remarkable that one does not need to directly follow the dynamics of the bath on the time scale of the system relaxation, in order to describe the latter. It should be noted that in cases involving slow relaxation, such as vibrational relaxation, this major simplification is absolutely crucial to one's ability to calculate the relaxation rate. ${ }^{18-23,43}$ Those observations reflect the ability of the memory kernel to focus on those features of the bath dynamics which actually influence the system. Unfortunately, the weak coupling approach also suffers from two serious shortcomings.

(1) The fact that $C(\tau)$ corresponds to the dynamics of the free bath indicates that, while the system is affected by the bath, the reverse action of the system on the bath remains unaccounted for. This observation highlights the limitations of the weak coupling approach, which cannot account for such phenomena as the reorganization of the solvent molecules around a solute following electron transfer or optical excitation.

(2) Since the bath typically consists of a huge number of DOF which are coupled via anharmonic potentials, the calculation of an exact quantum-mechanical correlation function, such as $C(\tau)$, is not computationally feasible (with the notable exception of harmonic baths). Thus, the calculation of the memory kernel has to rely on approximations, even in the limit of weak system-bath coupling.

\section{THE NAKAJIMA-ZWANZIG KERNEL IN TERMS OF SYSTEM-DEPENDENT BATH CORRELATION FUNCTIONS}

In this section, we present a new theoretical framework for calculating the Nakajima-Zwanzig memory kernel for an arbitrary system-bath coupling. For the sake of clarity, we state some of the main results without proof. The interested reader is referred to Appendix A for detailed derivations of those results.

The first step is to rewrite the Nakajima-Zwanzig memory kernel in the following form, which is completely equivalent to the standard form in Eq. (8) (cf. Appendix A): 


$$
\mathcal{K}(\tau)=\frac{1}{\hbar^{2}} \operatorname{Tr}_{b}\left\{\mathcal{L}_{\mathrm{bs}} e^{-i\left(\mathcal{L}-\mathcal{L}_{\mathrm{bs}} \mathcal{P}\right) \tau / \hbar} \mathcal{L}_{\mathrm{bs}} \hat{\rho}_{b}^{\mathrm{eq}}\right\} .
$$

We also note that (cf. Appendix A)

$$
\begin{aligned}
e^{-i\left(\mathcal{L}-\mathcal{L}_{\mathrm{bs}} \mathcal{P}\right) \tau / \hbar}= & e^{-i \mathcal{L} \tau / \hbar} \\
& +\frac{i}{\hbar} \int_{0}^{\tau} d \tau^{\prime} e^{-i \mathcal{L}\left(\tau-\tau^{\prime}\right) / \hbar} \mathcal{L}_{\mathrm{bs}} \mathcal{P} e^{-i\left(\mathcal{L}-\mathcal{L}_{\mathrm{bs}} \mathcal{P}\right) \tau^{\prime} / \hbar}
\end{aligned}
$$

Substituting Eq. (17) into Eq. (16) then yields the following identity:

$$
\mathcal{K}(\tau)=\mathcal{K}_{1}(\tau)+i \int_{0}^{\tau} d \tau^{\prime} \mathcal{K}_{1}\left(\tau-\tau^{\prime}\right) \mathcal{K}_{2}\left(\tau^{\prime}\right)
$$

where

$$
\mathcal{K}_{1}(\tau)=\frac{1}{\hbar^{2}} \operatorname{Tr}_{b}\left\{\mathcal{L}_{\mathrm{bs}} e^{-i \mathcal{L} \tau / \hbar} \mathcal{L}_{\mathrm{bs}} \hat{\rho}_{b}^{\mathrm{eq}}\right\}
$$

and

$$
\mathcal{K}_{2}(\tau)=\frac{1}{\hbar} \operatorname{Tr}_{b}\left\{e^{-i\left(\mathcal{L}-\mathcal{L}_{\mathrm{bs}} \mathcal{P}\right) \tau / \hbar} \mathcal{L}_{\mathrm{bs}} \hat{\rho}_{b}^{\mathrm{eq}}\right\} .
$$

We next substitute Eq. (17) into Eq. (20), which leads to the following integral equation for $\mathcal{K}_{2}(\tau)$ :

$$
\mathcal{K}_{2}(\tau)=\mathcal{K}_{3}(\tau)+i \int_{0}^{\tau} d \tau^{\prime} \mathcal{K}_{3}\left(\tau-\tau^{\prime}\right) \mathcal{K}_{2}\left(\tau^{\prime}\right),
$$

where

$$
\mathcal{K}_{3}(\tau)=\frac{1}{\hbar} \operatorname{Tr}_{b}\left\{e^{-i \mathcal{L} \tau / \hbar} \mathcal{L}_{\mathrm{bs}} \hat{\rho}_{b}^{\mathrm{eq}}\right\}
$$

Equations (18) and (21) represent the main result of this paper. They establish a closed form relationship between the sought after memory kernel, $\mathcal{K}(\tau)$, which is explicitly dependent on the projection operator $\mathcal{P}$, and $\mathcal{K}_{1}(\tau)$ and $\mathcal{K}_{3}(\tau)$, which are independent of it. It is important to note that $\mathcal{K}_{2}(\tau)$ is an auxiliary quantity in the sense that it can be obtained by solving Eq. (21), provided that $\mathcal{K}_{3}(\tau)$ is known. $\mathcal{K}_{2}(\tau)$ can then be substituted into Eq. (18), which is solved for $\mathcal{K}(\tau)$, provided that $\mathcal{K}_{1}(\tau)$ is known. Thus, finding $\mathcal{K}(\tau)$ translates into calculating $\mathcal{K}_{1}(\tau)$ and $\mathcal{K}_{3}(\tau)$, followed by solving Eq. (21) for $\mathcal{K}_{2}(\tau)$, and Eq. (18) for $\mathcal{K}(\tau)$.

Further insight into the significance of $\mathcal{K}_{1}(\tau), \mathcal{K}_{2}(\tau)$, and $\mathcal{K}_{3}(\tau)$ is obtained by considering their behavior in the limit of weak system-bath coupling. Thus, assuming that $\mathcal{L}_{\mathrm{bs}} \mathcal{P}$ and $\mathcal{L}_{\mathrm{bs}}$ in the exponents of Eqs. (20) and (22), respectively, can be neglected, one finds that $\mathcal{K}_{2}(\tau)$ and $\mathcal{K}_{3}(\tau)$ vanish

$$
\mathcal{K}_{2}(\tau) \approx \mathcal{K}_{3}(\tau) \approx \frac{1}{\hbar} \operatorname{Tr}_{b}\left\{e^{-i\left(\mathcal{L}_{s}+\mathcal{L}_{b}\right) \tau / \hbar} \mathcal{L}_{\mathrm{bs}} \hat{\rho}_{b}^{\mathrm{eq}}\right\}=0
$$

where the last equality in Eq. (23) is due to the fact that $\langle\hat{\Lambda}\rangle_{\mathrm{eq}}^{0}=0$. As a result,

$$
\mathcal{K}(\tau) \approx \mathcal{K}_{1}(\tau) \approx \frac{1}{\hbar^{2}} \operatorname{Tr}_{b}\left\{\mathcal{L}_{\mathrm{bs}} e^{-i\left(\mathcal{L}_{s}+\mathcal{L}_{b}\right) \tau / \hbar} \mathcal{L}_{\mathrm{bs}} \hat{\rho}_{b}^{\mathrm{eq}}\right\},
$$

which is the same as the weak coupling limit of the memory kernel, $\mathcal{K}_{\text {WC }}(\tau)$ [cf. Eq. (10)]. Thus, going beyond the weakcoupling limit will be manifested by shifting $\mathcal{K}(\tau)$ relative to $\mathcal{K}_{1}(\tau)$, as well as the creation of $\mathcal{K}_{2}(\tau)$ and $\mathcal{K}_{3}(\tau)$.

The main advantage of $\mathcal{K}_{1}(\tau)$ and $\mathcal{K}_{3}(\tau)$ over the original $\mathcal{K}(\tau)$, has to do with the fact that they do not contain the projection operator $\mathcal{P}$. However, even with this simplification, computing $\mathcal{K}_{1}(\tau)$ and $\mathcal{K}_{3}(\tau)$ is obviously not trivial, due to the real-time quantum dynamics of the overall system, embodied by $e^{-i \mathcal{L} \tau / \hbar}$. It is therefore important to estimate the computational cost of calculating $\mathcal{K}_{1}(\tau)$ and $\mathcal{K}_{3}(\tau)$ in practice. To this end, it is useful to rewrite the NakajimaZwanzig GQME in terms of the eigenrepresentation of $\hat{F}$ [cf. Eq. (2)]. Since $\hat{F}$ is most commonly given by a function of the system coordinates, we will assume below that the system position representation can be used for this purpose (the extension to other representations is straightforward). Thus, we assume that $\hat{F}=F(\hat{x})$, where $\hat{x}$ is the system position operator, which for the sake of simplicity will be assumed to be one dimensional, such that $F(\hat{x})|x\rangle=F(x)|x\rangle$ and $-\infty$ $<x<\infty$. We will also employ a Liouville space-based description and tetradic notation, ${ }^{57}$ where a system RDO is represented by a state vector in Liouville space

$$
\left\langle\left\langle x_{a}, x_{b} \mid \hat{\rho}_{s}\right\rangle\right\rangle \equiv \operatorname{Tr}_{s}\left[\left(\left|x_{a}\right\rangle\left\langle x_{b}\right|\right)^{\dagger} \hat{\rho}_{s}\right] \equiv\left\langle x_{a}\left|\hat{\rho}_{s}\right| x_{b}\right\rangle,
$$

and a system superoperator by a matrix

$$
\left\langle\left\langle x_{a}, x_{b}|\mathcal{K}| x_{1}, x_{2}\right\rangle\right\rangle \equiv \operatorname{Tr}_{s}\left[\left(\left|x_{a}\right\rangle\left\langle x_{b}\right|\right)^{\dagger} \mathcal{K}\left|x_{1}\right\rangle\left\langle x_{2}\right|\right] .
$$

The Nakajima-Zwanzig GQME, Eq. (7), and the integral equations, Eqs. (18) and (21), can then be rewritten in terms of the $x$ representation such that

$$
\begin{aligned}
\frac{d}{d t}\left\langle\left\langle x_{a}, x_{b} \mid \hat{\rho}_{s}\right\rangle\right\rangle & \\
= & -\frac{i}{\hbar} \int d x_{1} \int d x_{2}\left\langle\left\langle x_{a}, x_{b}\left|\mathcal{L}_{s}\right| x_{1}, x_{2}\right\rangle\right\rangle\left\langle\left\langle x_{1}, x_{2} \mid \hat{\rho}_{s}\right\rangle\right\rangle \\
& -\int_{0}^{t} d \tau \int d x_{1} \int d x_{2}\left\langle\left\langle x_{a}, x_{b}|\mathcal{K}(\tau)| x_{1}, x_{2}\right\rangle\right\rangle \\
& \times\left\langle\left\langle x_{1}, x_{2} \mid \hat{\rho}_{s}(t-\tau)\right\rangle\right\rangle, \\
\left\langle\left\langle x_{a},\right.\right. & \left.\left.x_{b}|\mathcal{K}(\tau)| x_{1}, x_{2}\right\rangle\right\rangle \\
= & \left\langle\left\langle x_{a}, x_{b}\left|\mathcal{K}_{1}(\tau)\right| x_{1}, x_{2}\right\rangle\right\rangle+i \int_{0}^{\tau} d \tau^{\prime} \int d x_{\mathrm{I}} \int d x_{\mathrm{II}} \\
& \times\left\langle\left\langle x_{a}, x_{b}\left|\mathcal{K}_{1}\left(\tau-\tau^{\prime}\right)\right| x_{\mathrm{I}}, x_{\mathrm{II}}\right\rangle\right\rangle\left\langle\left\langle x_{\mathrm{I}}, x_{\mathrm{II}}\left|\mathcal{K}_{2}\left(\tau^{\prime}\right)\right| x_{1}, x_{2}\right\rangle\right\rangle,
\end{aligned}
$$

and

$$
\begin{aligned}
\left\langle\left\langle x_{a}, x_{b}\left|\mathcal{K}_{2}(\tau)\right| x_{1}, x_{2}\right\rangle\right\rangle & \\
= & \left\langle\left\langle x_{a}, x_{b}\left|\mathcal{K}_{3}(\tau)\right| x_{1}, x_{2}\right\rangle\right\rangle+i \int_{0}^{\tau} d \tau^{\prime} \int d x_{\mathrm{I}} \int d x_{\mathrm{II}} \\
& \times\left\langle\left\langle x_{a}, x_{b}\left|\mathcal{K}_{3}\left(\tau-\tau^{\prime}\right)\right| x_{\mathrm{I}}, x_{\mathrm{II}}\right\rangle\right\rangle\left\langle\left\langle x_{\mathrm{I}}, x_{\mathrm{II}}\left|\mathcal{K}_{2}\left(\tau^{\prime}\right)\right| x_{1}, x_{2}\right\rangle\right\rangle .
\end{aligned}
$$


The corresponding matrix elements of $\mathcal{K}_{1}(\tau)$ and $\mathcal{K}_{3}(\tau)$ are explicitly given by

$$
\begin{aligned}
\left\langle\left\langle x_{a}, x_{b}\left|\mathcal{K}_{1}(\tau)\right| x_{1}, x_{2}\right\rangle\right\rangle & \\
= & \frac{1}{\hbar^{2}}\left\{F\left(x_{1}\right)\left[F\left(x_{a}\right)-F\left(x_{b}\right)\right]\langle\hat{\Lambda}(2, b, a, 1 ; \tau) \hat{\Lambda}\rangle_{\mathrm{eq}}^{0}\right. \\
& \left.\quad-F\left(x_{2}\right)\left[F\left(x_{a}\right)-F\left(x_{b}\right)\right]\langle\hat{\Lambda} \hat{\Lambda}(2, b, a, 1 ; \tau)\rangle_{\mathrm{eq}}^{0}\right\}
\end{aligned}
$$

and

$$
\begin{aligned}
\left\langle\left\langle x_{a}, x_{b}\left|\mathcal{K}_{3}(\tau)\right| x_{1}, x_{2}\right\rangle\right\rangle & \\
= & \frac{1}{\hbar}\left\{F\left(x_{1}\right)\left\langle\hat{I}_{b}(2, b, a, 1 ; \tau) \hat{\Lambda}\right\rangle_{\mathrm{eq}}^{0}\right. \\
& \left.-F\left(x_{2}\right)\left\langle\hat{\Lambda} \hat{I}_{b}(2, b, a, 1 ; \tau)\right\rangle_{\mathrm{eq}}^{0}\right\},
\end{aligned}
$$

where the average $\langle\cdots\rangle_{\mathrm{eq}}^{0}$ is the same as in Eq. (6) and

$$
\hat{\Gamma}(2, b, a, 1 ; \tau)=\left\langle x_{2}\left|e^{i \hat{H} \tau / \hbar}\right| x_{b}\right\rangle \hat{\Gamma}\left\langle x_{a}\left|e^{-i \hat{H} \tau / \hbar}\right| x_{1}\right\rangle .
$$

$\hat{\Gamma}$ is a bath operator, which in our case corresponds to either $\hat{\Lambda}$ or $\hat{I}_{b}$ (the latter is the unit operator in the bath Hilbert space).

It is important to note that all the information needed for determining the influence of the bath on the system is now contained in quantities of the form $\langle\hat{A}(2, b, a, 1 ; \tau) \hat{\Lambda}\rangle_{\text {eq }}^{0}$, where $\hat{A}$ is either $\hat{\Lambda}$ or $\hat{I}_{b}$. It should also be noted that those quantities satisfy the following time-reversal symmetry:

$$
\left[\langle\hat{B}(2, b, a, 1 ; \tau) \hat{A}\rangle_{\mathrm{eq}}^{0}\right]^{*}=\langle\hat{A} \hat{B}(1, a, b, 2 ; \tau)\rangle_{\mathrm{eq}}^{0},
$$

when $\hat{A}$ and $\hat{B}$ are Hermitian. We denote those quantities as system-dependent bath correlation functions (SDBCFs), for reasons that will become clear shortly. Although those SDBCFs are distinctly different from the standard free-bath correlation functions, $\langle\hat{A}(\tau) \hat{\Lambda}\rangle_{\mathrm{eq}}^{0}$ [e.g., Eq. (12)], they are directly related to them in the weak coupling limit. More specifically, $\hat{\Lambda}(2, b, a, 1 ; \tau)$ and $\hat{I}_{b}(2, b, a, 1 ; \tau)$ reduce into $\left\langle x_{2}\left|e^{i \hat{H}_{s} t / \hbar}\right| x_{b}\right\rangle\left\langle x_{a}\left|e^{-i \hat{H}_{s} t / \hbar}\right| x_{1}\right\rangle e^{i \hat{H}_{b} \tau / \hbar} \hat{\Lambda} e^{-i \hat{H}_{b} \tau / \hbar} \quad$ and $\left\langle x_{2}\left|e^{i \hat{H}_{s} t / \hbar}\right| x_{b}\right\rangle\left\langle x_{a}\left|e^{-i \hat{H}_{s} t / \hbar}\right| x_{1}\right\rangle \hat{I}_{b}$ in this limit, such that $\langle\hat{\Lambda} \hat{\Lambda}(2, b, a, 1 ; \tau)\rangle_{\mathrm{eq}}^{0}$ and $\langle\hat{\Lambda}(2, b, a, 1 ; \tau) \hat{\Lambda}\rangle_{\mathrm{eq}}^{0}$ turn into $\left\langle x_{2}\left|e^{i \hat{H}_{s} t / \hbar}\right| x_{b}\right\rangle\left\langle x_{a}\left|e^{-i \hat{H}_{s} t / \hbar}\right| x_{1}\right\rangle C(\tau) \quad$ and $\quad\left\langle x_{2}\left|e^{i \hat{H}_{s} t / \hbar}\right| x_{b}\right\rangle$ $\times\left\langle x_{a}\left|e^{-i \hat{H}_{s} t / \hbar}\right| x_{1}\right\rangle C(-\tau)$, respectively, and both $\left\langle\hat{I}_{b}(2, b, a, 1 ; \tau) \hat{\Lambda}\right\rangle_{\mathrm{eq}}^{0}$ and $\left\langle\hat{\Lambda} \hat{I}_{b}(2, b, a, 1 ; \tau)\right\rangle_{\mathrm{eq}}^{0}$ turn into $\left\langle x_{2}\left|e^{i \hat{H}_{s} t / \hbar}\right| x_{b}\right\rangle\left\langle x_{a}\left|e^{-i \hat{H}_{s} t / \hbar}\right| x_{1}\right\rangle\langle\hat{\Lambda}\rangle_{\mathrm{eq}}^{0}$, and vanish [in accord with the fact that $\mathcal{K}_{3}(\tau)=0$ in the weak coupling limit]. Replacing the FBCFs with SDBCFs signals the transition beyond the weak coupling limit, which must be accompanied by explicitly accounting for the reverse action of the system on the bath. This is accomplished in two ways: (1) the dynamics in the SDBCFs is determined by the overall Hamiltonian, $\hat{H}$, rather than the free bath Hamiltonian, $\hat{H}_{b}$ and (2) the propagators are "system-state selective," in the sense that they sum over system trajectories that are constrained to move forward from $x_{1}$ to $x_{a}$, and backward from $x_{b}$ to $x_{2}$.

It is important to note that, like FBCFs, SDBCFs are also expected to lose memory and decay to zero at times longer than a characteristic correlation time, i.e.,
$\langle\hat{A}(2, b, a, 1 ; \tau) \hat{\Lambda}\rangle_{\mathrm{eq}}^{0} \rightarrow\langle\hat{A}(2, b, a, 1 ; \tau)\rangle_{\mathrm{eq}}^{0} \times\langle\hat{\Lambda}\rangle_{\mathrm{eq}}^{0}=0$ at $\tau>\tau_{c}$. Furthermore, in many systems of practical interest, such as room temperature liquid solutions, the corresponding correlation time of the SDBCFs is expected to be relatively short. This implies that, as in the weak coupling limit, one does not need to directly follow the dynamics of the bath on the time scale of the system's relaxation, in order to describe the latter. It should also be noted that the cost of calculating a single SDBCF is comparable to that of computing a single FBCF, since the majority of the DOF accounted for in the overall Hamiltonian belong to the bath. Thus, at least roughly speaking, the computational cost of calculating the exact memory kernel is in fact comparable to that involved in calculating its weak coupling limit.

However, it should be emphasized that the weak coupling treatment also decouples the system dynamics from the bath dynamics, such that a single $\mathrm{FBCF}, C(\tau)$, is required in order to account for the effect of the bath on the system. This is no longer true beyond the weak coupling limit, since the SDBCFs entangle the DOF of the system and the bath. As a result, $\mathcal{K}(\tau)$, as well as $\mathcal{K}_{1}(\tau)$ and $\mathcal{K}_{3}(\tau)$, would generally be given in terms of a matrix with $N^{4}$ elements, in the case of an $N$ state system. The effective number of elements can be brought down somewhat, to $N^{3}(N-1) / 2$ in the case of $\mathcal{K}(\tau)$ and $\mathcal{K}_{1}(\tau)$, and $N^{2}\left(N^{2}+1\right) / 2$ in the case of $\mathcal{K}_{3}(\tau)$, by taking advantage of the following symmetries:

$\left\langle\left\langle x_{a}, x_{b}|\mathcal{K}(\tau)| x_{1}, x_{2}\right\rangle\right\rangle=\left\langle\left\langle x_{a}, x_{b}\left|\mathcal{K}_{1}(\tau)\right| x_{1}, x_{2}\right\rangle\right\rangle=0$ when $x_{a}=x_{b}$. This is clear from Eq. (30) in the case of $\mathcal{K}_{1}(\tau)$, and results from the fact that $\left|x_{a}\right\rangle\left\langle x_{a}\right|$ commutes with $\hat{F}$ in the case of $\mathcal{K}(\tau)$.

$\left\langle\left\langle x_{a}, x_{b}\left|\mathcal{K}_{1}(\tau)\right| x_{1}, x_{2}\right\rangle\right\rangle^{*}=\left\langle\left\langle x_{b}, x_{a}\left|\mathcal{K}_{1}(\tau)\right| x_{2}, x_{1}\right\rangle\right\rangle$, $\left\langle\left\langle x_{a}, x_{b}\left|\mathcal{K}_{3}(\tau)\right| x_{1}, x_{2}\right\rangle\right\rangle^{*}=\left\langle\left\langle x_{b}, x_{a}\left|\mathcal{K}_{3}(\tau)\right| x_{2}, x_{1}\right\rangle\right\rangle$, and $\left\langle\left\langle x_{a}, x_{b}|\mathcal{K}(\tau)| x_{1}, x_{2}\right\rangle\right\rangle^{*}=\left\langle\left\langle x_{b}, x_{a}|\mathcal{K}(\tau)| x_{2}, x_{1}\right\rangle\right\rangle$. This is clear from Eqs. (30) and (31) in the case of $\mathcal{K}_{1}(\tau)$ and $\mathcal{K}_{3}(\tau)$, and results from the fact that $\hat{\rho}_{s}$ must remain Hermitian throughout the time evolution in the case of $\mathcal{K}(\tau)$.

Thus, the computational effort required to generate the input required for computing the memory kernel is larger by a factor of about $N^{4} / 2$ in comparison to the weak coupling limit. Such scaling will obviously impose restrictions on actual applications. However, there is a very large number of applications that involve systems with a relatively small $N$, such as in electron transfer processes, optical spectroscopy, and vibrational relaxation, where the additional computational effort would be manageable.

The actual calculation of exact quantum mechanical correlation functions in a many-body system is obviously not generally feasible, regardless of whether it is a FBCF or a SDBCF. However, several strategies have been proposed over the last few decades in order to address the challenge of providing an effective, computationally feasible, and versatile approximate methods for calculating quantummechanical real-time correlation functions (cf. Sec. I). Many of those methods work better at short times, and will therefore be ideally suited for calculating the SDBCFs required for calculating the memory kernel. The combination of the 
theory presented herein with those methodologies will be reported elsewhere.

\section{APPLICATION TO A SYSTEM LINEARLY COUPLED TO A HARMONIC BATH}

In this section, we demonstrate the computational feasibility of calculating the Nakajima-Zwanzig memory kernel in the case of a system linearly coupled to a harmonic bath. The main advantage of this model has to do with the fact that the exact SDBCFs, and hence the memory kernel, can be computed in this case, via the QUAPI method of Makri and co-workers. ${ }^{10,134-146}$ However, we would like to emphasize that the approach is by no means restricted to this specific model, and that the memory kernel can also be computed in the case of nonlinear coupling to an anharmonic bath, provided that reasonable estimates of the SDBCFs are available.

The overall Hamiltonian for the model under consideration is given by

$$
\begin{aligned}
\hat{H}= & \hat{H}_{s}^{\prime}(\hat{x}) \\
& +\sum_{j}\left[\frac{\left(\hat{P}^{(j)}\right)^{2}}{2 M^{(j)}}+\frac{1}{2} M^{(j)}\left(\omega^{(j)}\right)^{2}\left(\hat{Q}^{(j)}-\frac{c^{(j)} F(\hat{x})}{M^{(j)}\left(\omega^{(j)}\right)^{2}}\right)^{2}\right],
\end{aligned}
$$

where $\left\{\hat{Q}^{(j)}\right\},\left\{\hat{P}^{(j)}\right\},\left\{\omega^{(j)}\right\}$, and $\left\{M^{(j)}\right\}$ are the coordinates, momenta, frequencies, and masses of the bath modes, and $\hat{H}_{s}^{\prime}(\hat{x})$ only depends on the system DOF. A complete characterization of the bath is provided by its spectral density function, defined by

$$
J(\omega)=\frac{\pi}{2} \sum_{j} \frac{\left(c^{(j)}\right)^{2}}{M^{(j)} \omega^{(j)}} \delta\left(\omega-\omega^{(j)}\right) .
$$

The system, bath, and system-bath terms, as defined in Eq. (1), are easily identified as

$$
\begin{aligned}
& \hat{H}_{s}(\hat{x})=\hat{H}_{s}^{\prime}(\hat{x})+\sum_{j} \frac{\left(c^{(j)}\right)^{2}}{2 M^{(j)}\left(\omega^{(j)}\right)^{2}} F^{2}(\hat{x}), \\
& \hat{H}_{b}=\sum_{j}\left[\frac{\left(\hat{P}^{(j)}\right)^{2}}{2 M^{(j)}}+\frac{1}{2} M^{(j)}\left(\omega^{(j)}\right)^{2}\left(\hat{Q}^{(j)}\right)^{2}\right] \equiv \sum_{j} \hat{H}_{b}^{(j)},
\end{aligned}
$$

and

$$
\hat{H}_{\mathrm{bs}}=\left[-\sum_{j} c^{(j)} \hat{Q}^{(j)}\right] \otimes F(\hat{x}) \equiv \hat{\Lambda} \otimes F(\hat{x}),
$$

where $\langle\hat{\Lambda}\rangle_{\text {eq }}^{0}=0$ is satisfied. We also define

$$
\begin{aligned}
\hat{H}_{\mathrm{env}}= & \sum_{j}\left[\frac{\left(\hat{P}^{(j)}\right)^{2}}{2 M^{(j)}}+\frac{1}{2} M^{(j)}\left(\omega^{(j)}\right)^{2}\right. \\
& \left.\times\left(\hat{Q}^{(j)}-\frac{c^{(j)} F(\hat{x})}{M^{(j)}\left(\omega^{(j)}\right)^{2}}\right)^{2}\right] \\
\equiv & \sum_{j} \hat{H}_{\mathrm{env}}^{(j)}
\end{aligned}
$$

for later use.

Consider first the $\operatorname{SDBCF}\left\langle\hat{I}_{b}(2, b, a, 1 ; \tau) \hat{\Lambda}\right\rangle_{\mathrm{eq}}^{0}$, which enters $\mathcal{K}_{3}(\tau)$ [cf. Eq. (31)]. Employing the quasiadiabatic splitting of $\hat{H}$ into $\hat{H}_{s}^{\prime}$ and $\hat{H}_{\mathrm{env}}$, one can put it in terms of the following path integral expression:

$$
\begin{aligned}
\left\langle\hat{I}_{b}(2, b, a, 1 ; \tau) \hat{\Lambda}\right\rangle_{\mathrm{eq}}^{0}= & \int d x_{1}^{+} \cdots \int d x_{N-1}^{+} \int d x_{N-1}^{-} \cdots \int d x_{1}^{-}\left\langle x_{N}^{+}\left|e^{-i \hat{H}_{s} \epsilon / \hbar}\right| x_{N-1}^{+}\right\rangle \cdots\left\langle x_{1}^{+}\left|e^{-i \hat{H}_{s} \epsilon / \hbar}\right| x_{0}^{+}\right\rangle \\
& \times\left\langle x_{0}^{-}\left|e^{i \hat{H}_{s} \epsilon / \hbar}\right| x_{1}^{-}\right\rangle \cdots\left\langle x_{N-1}^{-}\left|e^{i \hat{H}_{s} \epsilon / \hbar}\right| x_{N}^{-}\right\rangle F_{I}\left(x_{0}^{+}, \ldots, x_{N}^{+}, x_{N}^{-}, \ldots, x_{0}^{-}\right),
\end{aligned}
$$

where $\epsilon=\tau / N, x_{0}^{+}=x_{1}, x_{N}^{+}=x_{a}, x_{0}^{-}=x_{2}, x_{N}^{-}=x_{b}$,

$$
F_{I}\left(x_{0}^{+}, \ldots, x_{N}^{+}, x_{N}^{-}, \ldots, x_{0}^{-}\right)=\operatorname{Tr}_{b}\left[\hat{\rho}_{b}^{\mathrm{eq}} U_{\mathrm{env}}(0, \tau) U_{\mathrm{env}}(\tau, 0) \hat{\Lambda}\right]
$$

is the IF, and $U_{\text {env }}(\tau, 0)\left(U_{\text {env }}(0, \tau)\right)$ is the forward (backward) time evolution operator from time $0(\tau)$ to time $\tau(0)$, under the Hamiltonian $\hat{H}_{\text {env }}[x(t)]$, where $x(t)=\left\{x_{0}^{+}, \ldots, x_{N}^{+}\right\}\left(x(t)=\left\{x_{N}^{-}, \ldots, x_{0}^{-}\right\}\right)$.

$F_{I}\left(x_{0}^{+}, \ldots, x_{N}^{+}, x_{N}^{-}, \ldots, x_{0}^{-}\right)$, can be evaluated analytically. To this end, we note that

$$
\begin{aligned}
F_{I}\left(x_{0}^{+}, \ldots, x_{N}^{+}, x_{N}^{-}, \ldots, x_{0}^{-}\right) \propto & \operatorname{Tr}_{b}\left[e^{-\beta \hat{H}_{b}} U_{\mathrm{env}}(0, \tau) U_{\mathrm{env}}(\tau, 0) \hat{\Lambda}\right] \\
= & -\sum_{j} c^{(j)} \int d Q^{(j)} Q^{(j)}\left\langle Q^{(j)}\left|e^{-\beta \hat{H}_{b}^{(j)}} U_{\mathrm{env}}^{(j)}(0, \tau) U_{\mathrm{env}}^{(j)}(\tau, 0)\right| Q^{(j)}\right\rangle \\
& \times \prod_{k \neq j} \int d Q^{(k)}\left\langle Q^{(k)}\left|e^{-\beta \hat{H}_{b}^{(k)}} U_{\mathrm{env}}^{(k)}(0, \tau) U_{\mathrm{env}}^{(k)}(\tau, 0)\right| Q^{(k)}\right\rangle,
\end{aligned}
$$


and that $e^{-\beta \hat{H}_{b}^{(j)}} U_{\mathrm{env}}^{(j)}(0, \tau) U_{\mathrm{env}}^{(j)}(\tau, 0)$ can be considered as a single time evolution operator along a trajectory that consists of the following three segments:

(1) $0 \rightarrow \tau$ : Froward real-time propagation, under the Hamiltonian of a forced harmonic oscillator with driving force $F\left[x_{+}(t)\right]$, where $x_{+}(t)=\left\{x_{0}^{+}, \ldots, x_{N}^{+}\right\}$.

(2) $\tau \longrightarrow 0$ : Backward real-time propagation, under the Hamiltonian of a forced harmonic oscillator with driving force $F\left[x_{-}(t)\right]$, where $x_{-}(t)=\left\{x_{N}^{-}, \ldots, x_{0}^{-}\right\}$.

(3) $0 \rightarrow-i \hbar \beta$ : Imaginary-time propagation, under the Hamiltonian of a free harmonic oscillator.

The propagator of such a driven harmonic system is known in closed form, ${ }^{127}$ thereby enabling us to obtain an exact closed form expression for $F_{I}\left(x_{0}^{+}, \ldots, x_{N}^{+}, x_{N}^{-}, \ldots, x_{0}^{-}\right)$. A more detailed discussion of technical issues associated with this calculation is provided in Appendix B.

The remaining path integral over the system DOF, Eq.
(40), can be performed via direct numerical quadrature, provided that the number of states is not excessive and $\left\langle\hat{I}_{b}(2, b, a, 1 ; \tau) \hat{\Lambda}\right\rangle_{\text {eq }}^{0}$ is characterized by a relatively short correlation time. This is made possible by the quasiadiabatic splitting of $\hat{H}$ into $\hat{H}_{s}^{\prime}$ and $\hat{H}_{\text {env }}$, which leads to a relatively smooth integrand. For the same reason, the calculation can also be performed with relatively large time slices.

Next, consider the second type of SDBCF, $\langle\hat{\Lambda}(2, b, a, 1 ; \tau) \hat{\Lambda}\rangle_{\mathrm{eq}}^{0}$, which enters $\mathcal{K}_{1}(\tau)$ [cf. Eq. (30)]. Like $\left\langle\hat{I}_{b}(2, b, a, 1 ; \tau) \hat{\Lambda}\right\rangle_{\mathrm{eq}}^{0},\langle\hat{\Lambda}(2, b, a, 1 ; \tau) \hat{\Lambda}\rangle_{\mathrm{eq}}^{0}$ can also be written in terms of a path integral expression of the form of Eq. (40), but with a different IF

$$
\begin{aligned}
& F_{\Lambda}\left(x_{0}^{+}, \ldots, x_{N}^{+}, x_{N}^{-}, \ldots, x_{0}^{-}\right) \\
& \quad=\operatorname{Tr}_{b}\left[\hat{\rho}_{b}^{\mathrm{eq}} U_{\mathrm{env}}(0, \tau) \hat{\Lambda} U_{\mathrm{env}}(\tau, 0) \hat{\Lambda}\right]
\end{aligned}
$$

This IF can then be written in the following form:

$$
\begin{aligned}
F_{\Lambda}\left(x_{0}^{+}, \ldots, x_{N}^{+}, x_{N}^{-}, \ldots, x_{0}^{-}\right) \propto & \operatorname{Tr}_{b}\left[e^{-\beta \hat{H}_{b}} U_{\mathrm{env}}(0, \tau) \hat{\Lambda} U_{\mathrm{env}}(\tau, 0) \hat{\Lambda}\right] \\
= & \sum_{j, l} c^{(j)} c^{(l)} \int d Q^{(j)} \int d Q^{(l)} Q^{(j)} Q^{(l)}\left\langle Q^{(j)}\left|e^{-\beta \hat{H}_{b}^{(j)}} U_{\mathrm{env}}^{(j)}(0, \tau)\right| Q^{(l)}\right\rangle \\
& \times\left\langle Q^{(l)}\left|U_{\mathrm{env}}^{(j)}(\tau, 0)\right| Q^{(j)}\right\rangle \prod_{k \neq j, l} \int d Q^{(k)}\left\langle Q^{(k)}\left|e^{-\beta \hat{H}_{b}^{(k)}} U_{\mathrm{env}}^{(k)}(0, \tau) U_{\mathrm{env}}^{(k)}(\tau, 0)\right| Q^{(k)}\right\rangle .
\end{aligned}
$$

In this case, we need to treat two propagators, rather than a single one, namely $\left\langle Q^{(j)}\left|e^{-\beta \hat{H}_{b}^{(j)}} U_{\mathrm{env}}^{(j)}(0, \tau)\right| Q^{(l)}\right\rangle$ and $\left\langle Q^{(l)}\left|U_{\text {env }}^{(j)}(\tau, 0)\right| Q^{(j)}\right\rangle$, each of which can be evaluated in closed form. The remaining double Gaussian integral over $Q^{(j)}$ and $Q^{(l)}$ is somewhat cumbersome, but otherwise straightforward, and leads to an exact closed form expression for $F_{\Lambda}\left(x_{0}^{+}, \ldots, x_{N}^{+}, x_{N}^{-}, \ldots, x_{0}^{-}\right)$. The double Gaussian integrals which are used in the evaluation of those terms are given in Appendix C.

As an example, we present the results obtained via the approach discussed above, in the case of a two-level system with the following Hamiltonian:

$$
\hat{H}_{s}=\Omega \hat{\sigma}_{x}+\epsilon \hat{\sigma}_{z},
$$

which is coupled to the harmonic bath via the operator $F(\hat{x})=\hat{x}=\hat{\sigma}_{z}$. Here, $\hat{\sigma}_{x}=|+\rangle\langle-|+|-\rangle\langle+|$ and $\hat{\sigma}_{z}=|+\rangle\langle+|-|-\rangle$ $\times\langle-|$, where $\hat{\sigma}_{z}| \pm\rangle= \pm| \pm\rangle$. It should be noted that $\hat{\sigma}_{z}$ plays the role of the system coordinate, $\hat{x}$, in this case, such that the system can be described on a two-point grid, and the spatial integral, $\int d x$, turns into a sum over those two states, $\Sigma_{j= \pm}$. We also note that $\hat{H}_{s}^{\prime}=\hat{H}_{s}$ in this case, since $F^{2}(\hat{x}) \equiv \hat{\sigma}_{z}^{2}=\hat{I}_{s}$ is constant.

The superoperator $\mathcal{K}(\tau)$ is represented by a $4 \times 4$ matrix in terms of the $\{|++\rangle\rangle,|+-\rangle\rangle,|-+\rangle\rangle,|--\rangle\rangle\}$ representation. However, as noted in Sec. III, $\left\langle\left\langle++|\mathcal{K}(\tau)| x_{1} x_{2}\right\rangle\right\rangle=\left\langle\left\langle--|\mathcal{K}(\tau)| x_{1} x_{2}\right\rangle\right\rangle=0$, and $\left\langle\left\langle+-|\mathcal{K}(\tau)| x_{1} x_{2}\right\rangle\right\rangle=\left\langle\left\langle-+|\mathcal{K}(\tau)| x_{2} x_{1}\right\rangle\right\rangle^{*}$, such that there are only four independent matrix elements, which will be chosen as $\langle\langle+-|\mathcal{K}(\tau)|++\rangle\rangle,\langle\langle+-|\mathcal{K}(\tau)|--\rangle\rangle$, $\langle\langle+-|\mathcal{K}(\tau)|+-\rangle\rangle$, and $\langle\langle+-|\mathcal{K}(\tau)|-+\rangle\rangle$. Those elements are also compared to their counterparts at the weak coupling limit. The latter can be obtained explicitly, and anticipating the following application, are given below for the case where $\epsilon=\Omega$ :

$$
\begin{aligned}
& \left\langle\left\langle+-\left|\mathcal{K}_{\mathrm{WC}}(\tau)\right|++\right\rangle=-\frac{2 \sqrt{2}}{\hbar^{2}} \sin (2 \Omega \tau)\left[\cos (2 \Omega \tau)-\frac{i}{\sqrt{2}} \sin (2 \Omega \tau)\right] \operatorname{Im} C(\tau)\right. \\
& \left\langle\left\langle+-\left|\mathcal{K}_{\mathrm{WC}}(\tau)\right|--\right\rangle=\left\langle\left\langle+-\left|\mathcal{K}_{\mathrm{WC}}(\tau)\right|++\right\rangle\right\rangle\right. \\
& \left\langle\left\langle+-\left|\mathcal{K}_{\mathrm{WC}}(\tau)\right|-+\right\rangle=-\frac{2}{\hbar^{2}} \sin ^{2}(2 \Omega \tau) \operatorname{Re} C(\tau)\right. \\
& \left\langle\left\langle+-\left|\mathcal{K}_{\mathrm{WC}}(\tau)\right|+-\right\rangle=\frac{4}{\hbar^{2}}\left[\cos (2 \Omega \tau)-\frac{i}{\sqrt{2}} \sin (2 \Omega \tau)\right]^{2} \operatorname{Re} C(\tau) .\right.
\end{aligned}
$$




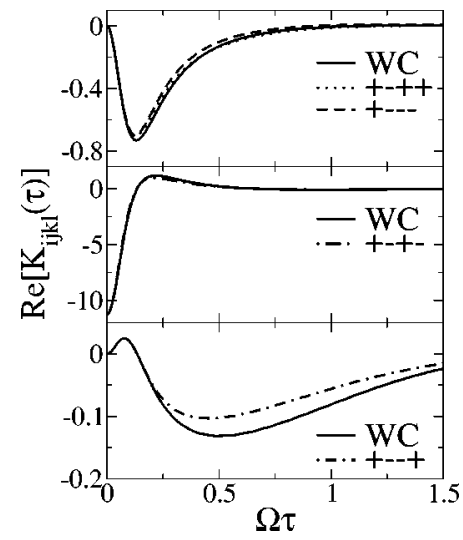

FIG. 1. The real parts of $\langle\langle+-|\mathcal{K}(\tau)|++\rangle\rangle$ and $\langle\langle+-|\mathcal{K}(\tau)|--\rangle\rangle$ (upper panel), $\langle\langle+-|\mathcal{K}(\tau)|+-\rangle\rangle$ (middle panel), and $\langle\langle+-|\mathcal{K}(\tau)|-+\rangle\rangle$ (lower panel). Also shown are the corresponding predictions in the weak coupling (WC) limit (solid lines). Note that $\langle\langle+-|\mathcal{K}(\tau)|++\rangle\rangle$ $=\langle\langle+-|\mathcal{K}(\tau)|--\rangle\rangle$ at the WC limit.

The results reported below were obtained for $\epsilon=\Omega$ $=1.0, \beta \hbar \Omega=5.0$,

$$
J(\omega)=\xi \frac{\pi}{2} \omega e^{-\omega / \omega_{c}}
$$

$\xi=0.1$ and $\omega_{c} / \Omega=7.5 . \mathcal{K}_{1}(\tau)$ and $\mathcal{K}_{3}(\tau)$ have been obtained by dividing each value of $\tau$ into 10 or less equal segments, and calculating the corresponding path integrals via direct multiple summation. Each element of $\mathcal{K}_{1}(\tau)$ and $\mathcal{K}_{3}(\tau)$ has been calculated on a 300 point time grid with a time step of $0.01 \Omega^{-1}$. Equation (21) has been solved for $\mathcal{K}_{2}(\tau)$, via an iterative procedure, with $\mathcal{K}_{2}(\tau)=\mathcal{K}_{3}(\tau)$ as the initial guess (10-20 iterations were required for convergence). The resulting $\mathcal{K}_{2}(\tau)$ was then substituted into Eq. (18), so as to obtain $\mathcal{K}(\tau)$ on the same 300 point time grid. The matrix representing $\mathcal{K}(\tau)$ has been kept in memory throughout the subsequent numerical solution of the GQME, which was carried out by the second-order Runge-Kutta method. ${ }^{203}$ The same time step of $0.01 \Omega^{-1}$ has been used, and $\hat{\rho}_{s}$ over the previous 300 steps has been kept in memory in order to evaluate the time derivative.

The real and imaginary parts of $\langle\langle+-|\mathcal{K}(\tau)|++\rangle\rangle$, $\langle\langle+-|\mathcal{K}(\tau)|--\rangle\rangle,\langle\langle+-|\mathcal{K}(\tau)|+-\rangle\rangle$, and $\langle\langle+-|\mathcal{K}(\tau)|-$ $+\rangle\rangle$ are shown in Figs. 1 and 2, respectively. A close inspec-

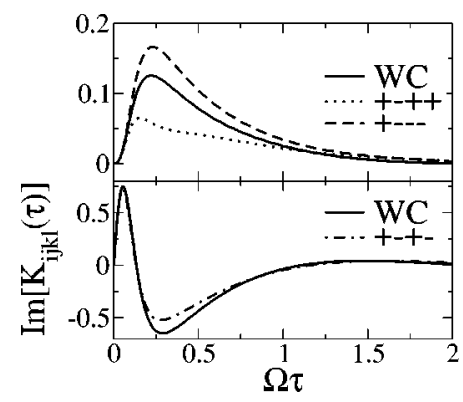

FIG. 2. The imaginary parts of $\langle\langle+-|\mathcal{K}(\tau)|++\rangle\rangle$ and $\langle\langle+-|\mathcal{K}(\tau)|$ $--\rangle\rangle$ (upper panel), and $\langle\langle+-|\mathcal{K}(\tau)|+-\rangle\rangle$ (lower panel). Note that $\operatorname{Im}\langle\langle+-|\mathcal{K}(\tau)|-+\rangle\rangle=0$ in this case (see lower panel of Fig. 1 for its real part). Also shown are the corresponding predictions in the WC limit (solid lines). Note that $\langle\langle+-|\mathcal{K}(\tau)|++\rangle\rangle=\langle\langle+-|\mathcal{K}(\tau)|--\rangle\rangle$ at the WC limit.

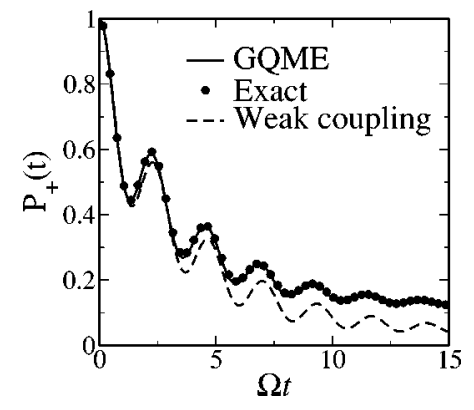

FIG. 3. The relaxation of $P_{+}(t)=\left\langle+\left|\hat{\rho}_{s}(t)\right|+\right\rangle$ to equilibrium. Shown are the predictions of the Nakajima-Zwanzig GQME (solid line), the exact result as obtained via the iterative tensor propagator method of Makri et al. (solid circles), and a prediction based on the WC treatment (dashed line).

tion of those figures lead to the following observations:

(1) All four elements decay to zero at long times, with correlation times which are comparable to that of $C(\tau)$.

(2) With the exception of $\langle\langle+-|\mathcal{K}(\tau)|+-\rangle\rangle$, all elements vanish at $\tau=0$. This is consistent with the explicit expressions we obtained for the weak coupling limit, Eq. (46), and appears to remain valid beyond it.

(3) The equivalence of $\langle\langle+-|\mathcal{K}(\tau)|++\rangle\rangle$ and $\langle\langle+-|\mathcal{K}(\tau)|--\rangle\rangle$ is not satisfied beyond the weak coupling limit. The imaginary part is observed to be particularly sensitive to the departure from the weak coupling limit, with $\operatorname{Im}\langle\langle+-|\mathcal{K}(\tau)|++\rangle\rangle$ and $\operatorname{Im}\langle\langle+-|\mathcal{K}(\tau)|--\rangle\rangle$ significantly lower and higher, respectively, relative to the weak coupling result.

In Fig. 3, we show the relaxation of the population of state $|+\rangle, P_{+}(t)=\left\langle+\left|\hat{\rho}_{s}(t)\right|+\right\rangle$, to equilibrium, starting from the initial state $\hat{\rho}_{s}(0)=|+\rangle\langle+|$. Shown are the numerically exact result, as obtained from a direct implementation of the iterative tensor QUAPI method of Makri et al., ${ }^{10,134-146}$ the result obtained via solving the NakajimaZwanzig GQME, and the prediction of the weak coupling treatment. The fact that the asymptotic equilibrium value of $P_{+}$clearly deviates from the weak coupling prediction, indicates that the system-bath coupling cannot be treated as weak in this case. The fact that the GQME-based result essentially coincides with the exact result is reassuring, and demonstrates the ability of the GQME-based method to give an accurate description of dissipative quantum dynamics beyond the weak coupling limit. It should be noted that both the exact and GQME-based method use the QUAPI method for calculating the dynamics over short time segments, in order to generate the dynamics on a longer time scale. In the case of the GQME approach, this short time segment is naturally defined as the correlation time of the SDBCFs, which is about 1 order of magnitude shorter than the actual lifetime of $P_{+}$(compare the time axes of Fig. 3 and Figs. 1 and 2). The same time represents the range of the nonlocal coupling between paths in the case of the iterative tensor propagator method, which is dictated by the influence functional. We will compare the scaling of those two ways of accounting for the finite range of the bath memory in the next Sec. V. 


\section{CONCLUSIONS}

The proposed GQME-based approach offers several important advantages for simulating quantum dynamics in condensed-phase systems:

(1) The validity of the Nakajima-Zwanzig GQME is not restricted to Markovian dynamics in the limit of weak system-bath coupling, as in the case of standard Markovian QMEs, or to systems which are in the close vicinity of equilibrium, as in the case of LRT. As a result, it can account for the quantum dynamics of a system, which is strongly coupled to an anharmonic environment, and possibly subject to a strong time-dependent perturbation. For example, it can be used to describe the response to an intense laser pulse, of a polar solute molecule embedded in a polar liquid solvent.

(2) The RDO provides a complete description of the state of the system. Thus, one can now calculate all observables of interest from a single simulation (e.g., one can obtain the phase and population relaxation at the same time). Furthermore, rare event statistics cease to be a problem, because the RDO automatically accounts for complete ensemble averaging. For example, one can obtain a barrier crossing rate constant from a direct and short nonequilibrium simulation, without resorting to the reactive flux method (the length of the simulation is determined by the time it takes the system to establish rate kinetics, rather than by the very slow time scale set by the rate constant for barrier crossing).

(3) The input regarding the bath dynamics is kept to a minimum via the memory kernel, which essentially filters out those aspects of the bath dynamics that the system is sensitive to. It is important to note that our methodology for obtaining the memory kernel is completely general, and is by no means restricted to harmonic systems. The accuracy of the result obviously depends on that of the SDBCFs, which serve as input, and numerically exact SDBCFs can only be obtained in the case of systems linearly coupled to a harmonic bath (e.g., via the QUAPI method). However, one can easily envision calculating those SDBCFs with the help of approximate schemes, such as those provided by SC and analytical continuation methods. It is important to note that many of those techniques are restricted to calculating correlation functions, or give predictions of deteriorating quality at longer times when used to directly simulate nonequilibrium dynamics. Thus, one expects to obtain significantly more reliable predictions by restricting the use of those methods to calculating the SDBCFs, which are often relatively short lived, and solving the Nakajima-Zwanzig GQME in a numerically exact manner, which ought to be feasible due to the small size of the system.

(4) It is important to note that our formulation puts the memory kernel in terms of two-time, rather than multitime, SDBCFs. Previous attempts to go beyond the limit of weak system-bath coupling by including higher order terms in the perturbation expansion, involve multidimensional time integrals over free-bath multitime correlation functions (e.g., four-time correlation functions in the case of the fourth-order QME). ${ }^{104,116-119}$ This appears to be the price one has to pay for using a zero Hamiltonian which does not include the system-bath coupling. A similar type of quantity shows up when one goes beyond LRT, ${ }^{57,150}$ in order to account for nonlinear response functions (e.g., in the theory of four-wave mixing experiments, which can be attributed to the fact that the zero order Hamiltonian does not include the field-matter interaction). Such multitime correlation functions are notoriously difficult to compute even from classical MD simulations. Our approach seems to avoid them because of its inherently nonperturbative nature, which leads to an equivalent formulation in terms of two-time system-dependent, as opposed to free-bath, correlation functions.

The new approach is similar in spirit to the path integral IF approach. Like the IF, the memory kernel provides a formally exact compact parameterization of the influence of the bath on the system dynamics. Particularly relevant for comparison is the IF-based iterative tensor propagator method of Makri and co-workers, ${ }^{138-141,143,145}$ which is based on using the IF for the time interval that corresponds to the correlation time. Furthermore, one can use path integral IF techniques in order to compute the SDBCFs, which provide the input for the calculation of the memory kernel (e.g., see our calculation of the SDBCFs in the case of linear coupling to a harmonic bath in Sec. IV). However, despite those similarities, the GQME-based approach appears to have several important advantages over the full blown IF approach.

(1) The GQME-based approach provides a more favorable "packaging" of the information regarding the influence of the bath over the system's dynamics. More specifically, if $N$ is the number of states (e.g., corresponding to the states included in the discrete variable representation), and $k_{\max }$ is the number of time slices that the correlation time is divided into, then the storage of the IF scales like $(2 N)^{2 k_{\max }}$ (within the iterative tensor propagator method of Makri and co-workers ${ }^{138-141,143,145}$ ), while that of the memory kernel scales like $\sim N^{4} k_{\max }$. This favorable scaling is intimately related to the fact that the memory kernel can be expressed in terms of $\sim N^{4}$ two-time correlation functions, whereas the IF is a function of the $2 k_{\max }$ variables that correspond to the discrete representation of the system's path. Thus, while it is relatively straightforward to store the memory kernel of a system with relatively large $N$ and $k_{\max }$ in memory throughout the simulation, this is not the case for the IF. The recent attempt by Golosov et al. to improve the scaling of the iterative tensor method, so as to make it go like $\sim N^{4} k_{\max }$, instead of $(2 N)^{2 k_{\max }}$, is interesting to note in this respect. ${ }^{147,148}$ However, it should be emphasized that their "memory equation algorithm" involves approximations, which have led to noticeable deviations when tested against benchmarks, whereas our approach is exact (for example, see the comparison to a benchmark in Fig. 3).

(2) The structure of the IF approach forces a description of the system dynamics in terms of path integrals. However, this may not be the most cost-effective framework for simulating the dynamics of what is usually a relatively small quantum system. At the same time, the description of the system dynamics via the GQME is formulated in terms of operators, and is therefore free of such constraints. Thus, once the memory kernel is known, either with or without the help of a path-integral-based methodology, one is free to choose the most optimal numerical technique for solving the GQME. 
The new formulation of the Nakajima-Zwanzig memory kernel in terms of SDBCFs developed herein, combined with recent advances in techniques for calculating quantummechanical correlation functions, and the distinct advantages of a methodology based on the Nakajima-Zwanzig GQME, open the door to many exciting applications that would have been difficult to pursue otherwise. Such applications include electronic and vibrational relaxation, chromophore spectroscopy, and chemical reactivity, in solution. Those and other applications are the subject of ongoing work in our group, and will be reported in future publications.

\section{ACKNOWLEDGMENT}

This project was supported by the National Science Foundation FOCUS Center, through Grant No. PHY0114336.

\section{APPENDIX A: REFORMULATION OF THE NAKAJIMA-ZWANZIG KERNEL}

In this Appendix, we provide the detailed proofs of some of the results that were utilized in Sec. III. We start by proving that Eq. (16) is equivalent to Eq. (8). The proof is based on the following identity:

$$
\mathcal{Q} \mathcal{L} \mathcal{Q}=\mathcal{Q}\left(\mathcal{L}-\mathcal{L}_{\text {bs }} \mathcal{P}\right)
$$

The proof of Eq. (A1) is based on the following observations: (1) $\mathcal{Q} \mathcal{L}_{s} \mathcal{Q}=\mathcal{Q} \mathcal{L}_{s}$, since $\mathcal{P} \mathcal{L}_{s} \mathcal{P}=\mathcal{L}_{s} \mathcal{P}^{2}=\mathcal{L}_{s} \mathcal{P}$; (2) $\mathcal{Q} \mathcal{L}_{b} \mathcal{Q}=\mathcal{Q} \mathcal{L}_{b}$, since $\left[\hat{H}_{b}, \hat{\rho}_{b}^{\mathrm{eq}}\right]=0 ; \quad(3) \quad \mathcal{Q} \mathcal{L}_{\mathrm{bs}} \mathcal{Q}=\mathcal{Q}\left(\mathcal{L}_{\mathrm{bs}}\right.$ $-\mathcal{L}_{\text {bs }} \mathcal{P}$ ). One can then show that $e^{-i \mathcal{Q} \mathcal{L} \tau / \hbar} \mathcal{Q}$ $=\mathcal{Q} e^{-i\left(\mathcal{L}-\mathcal{L}_{\mathrm{bs}} \mathcal{P}\right) \tau / \hbar}:$

$$
\begin{aligned}
e^{-i \mathcal{Q} \mathcal{L} / \hbar} \mathcal{Q}= & {\left[\hat{I}-\frac{i}{\hbar} \mathcal{Q} \mathcal{L} \tau-\frac{1}{2 \hbar^{2}} \mathcal{Q} \mathcal{L} \mathcal{Q} \mathcal{L} \tau^{2}+\ldots\right] \mathcal{Q} } \\
= & \mathcal{Q}-\frac{i}{\hbar} \mathcal{Q} \mathcal{L} \mathcal{Q} \tau-\frac{1}{2 \hbar^{2}}(\mathcal{Q} \mathcal{L} \mathcal{Q})^{2} \tau^{2}+\cdots \\
= & \mathcal{Q}-\frac{i}{\hbar} \mathcal{Q}\left(\mathcal{L}-\mathcal{L}_{\mathrm{bs}} \mathcal{P}\right) \tau-\frac{1}{2 \hbar^{2}} \\
& \times\left[\mathcal{Q}\left(\mathcal{L}-\mathcal{L}_{\mathrm{bs}} \mathcal{P}\right)\right]^{2} \tau^{2}+\cdots \\
= & \mathcal{Q}\left[\hat{I}-\frac{i}{\hbar}\left(\mathcal{L}-\mathcal{L}_{\mathrm{bs}} \mathcal{P}\right) \tau-\frac{1}{2 \hbar^{2}}\left(\mathcal{L}-\mathcal{L}_{\mathrm{bs}} \mathcal{P}\right)^{2} \tau^{2}\right] \\
= & \mathcal{Q} e^{-i\left(\mathcal{L}-\mathcal{L}_{\mathrm{bs}} \mathcal{P}\right) \tau / \hbar},
\end{aligned}
$$

where the second equality is based on the identity $\mathcal{Q}^{2}=\mathcal{Q}$, the third is based on Eq. (A1), and the fourth on the identity $\mathcal{P} \mathcal{Q}=0$. Substituting $\mathcal{Q} e^{-i\left(\mathcal{L}-\mathcal{L}_{\mathrm{bs}} \mathcal{P}\right) \tau / \hbar}$ for $e^{-i \mathcal{Q} \mathcal{L} \tau / \hbar} \mathcal{Q}$ in Eq. (8), and noting that $\operatorname{Tr}_{b}\left[\mathcal{L}_{\text {bs }} \mathcal{Q}\right]=\operatorname{Tr}_{b}\left[\mathcal{L}_{\text {bs }}\right]$, which is in turn based on the fact that $\langle\hat{\Lambda}\rangle_{\text {eq }}^{0}=0$, leads to Eq. (16).

We next prove the identity in Eq. (17). To this end, consider the following time derivative:

$$
\frac{d}{d \tau}\left[e^{i \mathcal{L} \tau / \hbar} e^{-i\left(\mathcal{L}-\mathcal{L}_{\mathrm{bs}} \mathcal{P}\right) \tau / \hbar}\right]=\frac{i}{\hbar} e^{i \mathcal{L} \tau / \hbar} \mathcal{L}_{\mathrm{bs}} \mathcal{P} e^{-i\left(\mathcal{L}-\mathcal{L}_{\mathrm{bs}} \mathcal{P}\right) \tau / \hbar} .
$$

The identity in Eq. (17) can be obtained by integrating both sides of Eq. (A3) from 0 to $\tau$, followed by multiplication by $e^{-i \mathcal{L} \tau / \hbar}$ from the left.

Finally, we note that Eqs. (18) and (21) are obtained by a straightforward substitution of the identity in Eq. (17) into Eqs. (16) and (20), respectively, and noting that $\operatorname{Tr}_{b}[\hat{A} \mathcal{P} \hat{B}]$ $=\operatorname{Tr}_{b}\left[\hat{A} \hat{\rho}_{b}^{\mathrm{eq}}\right] \operatorname{Tr}_{b}[\hat{B}]$.

\section{APPENDIX B: THE CALCULATION OF $F_{l}\left(x_{0}^{+}, \ldots, x_{N}^{+}, x_{N}^{-}, \ldots, x_{0}^{-}\right)$}

The key to calculating $F_{I}\left(x_{0}^{+}, \ldots, x_{N}^{+}, x_{N}^{-}, \ldots, x_{0}^{-}\right)$is the evaluation of $\left\langle Q^{(j)}\left|e^{-\beta \hat{H}_{b}^{(j)}} U_{\mathrm{env}}^{(j)}(0, \tau) U_{\mathrm{env}}^{(j)}(\tau, 0)\right| Q^{(j)}\right\rangle$ [cf. Eq. (42)]. The latter can be obtained analytically in closed form (for the sake of clarity, we drop the index $j$ from this point on) ${ }^{127}$

$$
\begin{aligned}
& \left\langle Q\left|e^{-\beta \hat{H}_{b}} U_{\text {env }}(0, \tau) U_{\text {env }}(\tau, 0)\right| Q\right\rangle \\
& =\left[\frac{M \omega}{2 \pi \hbar \sinh (\beta \hbar \omega)}\right]^{1 / 2} e^{-A Q^{2}+B Q+C},
\end{aligned}
$$

where

$$
\begin{aligned}
A= & \frac{M \omega}{\hbar \sinh (\beta \hbar \omega)}[\cosh (\beta \hbar \omega)-1], \\
B= & \frac{i c}{\hbar \sinh (\beta \hbar \omega)} \int_{\Gamma} d t^{\prime} F\left(t^{\prime}\right) \\
& \times\left[\sin \left(\omega t^{\prime}\right)-\sin \left(\omega\left(t^{\prime}+i \hbar \beta\right)\right)\right],
\end{aligned}
$$

and

$$
\begin{aligned}
C= & -\frac{i c^{2}}{\hbar \omega \sinh (\beta \hbar \omega)} \int_{\Gamma} d t^{\prime} \int_{\Gamma^{\prime}\left(t^{\prime}\right)} d t^{\prime \prime} F\left(t^{\prime}\right) F\left(t^{\prime \prime}\right) \\
& \times \sin \left(\omega\left(t^{\prime}+i \hbar \beta\right)\right) \sin \left(\omega t^{\prime \prime}\right)-\frac{i c^{2}}{2 M \omega^{2}} \int_{\Gamma} d t^{\prime} F^{2}\left(t^{\prime}\right) .
\end{aligned}
$$

Here, $\Gamma$ corresponds to the integration over the following contour in the complex plane: $0 \rightarrow t \rightarrow 0 \rightarrow-i \hbar \beta, \Gamma^{\prime}\left(t^{\prime}\right)$ is its segment that ends at $t^{\prime}$, and $F(t)=F[x(t)]$, such that

$$
x(t)=\left\{\begin{array}{ll}
x_{+}(t) & \text { for } 0 \rightarrow t \\
x_{-}(t) & \text { for } t \rightarrow 0 \\
0 & \text { for } 0 \rightarrow-i \hbar \beta
\end{array} .\right.
$$

The remaining integrals over $Q^{(k)}$ in Eq. (42) are Gaussian, and can be performed analytically based on the following well known identities:

$$
\begin{aligned}
& \int_{-\infty}^{\infty} d y y e^{-a y^{2}+b y}=\sqrt{\frac{\pi}{a}} \frac{b}{2 a} e^{b^{2} / 4 a}, \\
& \int_{-\infty}^{\infty} d y e^{-a y^{2}+b y}=\sqrt{\frac{\pi}{a}} e^{b^{2} / 4 a} .
\end{aligned}
$$

In the actual calculation, $x(t)$ is discretized, such that $x(t) \rightarrow\left(x_{0}^{+}, \ldots, x_{N}^{+}, x_{N}^{-}, \ldots, x_{0}^{-}\right) \equiv\left(x_{0} \ldots x_{2 N+1}\right)$. Since $F(t)$ has a fixed value in each time slice, one can perform the 
corresponding time integrals in the coefficients $B$ and $C$ analytically. This leads to the following working expressions for $B$ and $C$ :

$$
\begin{aligned}
B= & \frac{i c}{\hbar \omega \sinh (\beta \hbar \omega)} \sum_{j=0}^{2 N} F\left(x_{j}\right)\left[\cos \left(\omega t_{j}\right)-\cos \left(\omega\left(t_{j+1}\right)\right)-\cos \left(\omega\left(t_{j}+i \hbar \beta\right)\right)+\cos \left(\omega\left(t_{j+1}+i \hbar \beta\right)\right)\right], \\
C= & -\frac{i c^{2}}{\hbar \omega \sinh (\beta \hbar \omega)}\left\{\sum_{j=1}^{2 N+1} \sum_{k=0}^{j-1} \frac{F\left(x_{j}\right) F\left(x_{k}\right)}{\omega^{2}}\left[\cos \left(\omega\left(t_{j+1}+i \hbar \beta\right)\right)-\cos \left(\omega\left(t_{j}+i \hbar \beta\right)\right)\right]\left[\cos \left(\omega t_{k}\right)-\cos \left(\omega t_{k+1}\right)\right]\right. \\
& +\sum_{j=1}^{2 N+1} \frac{F^{2}\left(x_{j}\right)}{\omega^{2}}\left(\cos \left(\omega t_{j}\right)\left[\cos \left(\omega\left(t_{j+1}+i \hbar \beta\right)\right)-\cos \left(\omega\left(t_{j}+i \hbar \beta\right)\right)\right]\right. \\
& \left.-\frac{1}{4}\left[\cos \left(\omega\left(2 t_{j+1}+i \hbar \beta\right)\right)-\cos \left(\omega\left(2 t_{j}+i \hbar \beta\right)\right)\right)\right\} .
\end{aligned}
$$

\section{APPENDIX C: INTEGRALS USED IN THE EVALUATION OF $\langle\hat{\Lambda}(2, b, a, 1 ; \tau) \hat{\Lambda}\rangle_{\mathrm{eq}}^{0}$}

The following integrals are useful for evaluating $F_{\Lambda}\left(x_{0}^{+}, \ldots, x_{N}^{+}, x_{N}^{-}, \ldots, x_{0}^{-}\right)$in Eq. (44):

$$
\begin{aligned}
& \int_{-\infty}^{\infty} d X_{A} \int_{-\infty}^{\infty} d X_{B} \exp \left[-A\left(X_{A}^{2}+X_{B}^{2}\right)-B X_{A} X_{B}-C X_{A}-D X_{B}\right] X_{A} X_{B} \\
& =\frac{B^{2}(B+C D)+2 A B\left(C^{2}+D^{2}\right)-4 A^{2}(B-C D)}{\left(4 A^{2}-B^{2}\right)^{2}} \frac{2 \pi}{\sqrt{4 A^{2}-B^{2}}} \exp \left\{-\frac{B C D-A\left(C^{2}+D^{2}\right)}{4 A^{2}-B^{2}}\right\} \\
& \int_{-\infty}^{\infty} d X_{A} \int_{-\infty}^{\infty} d X_{B} \exp \left[-A\left(X_{A}^{2}+X_{B}^{2}\right)-B X_{A} X_{B}-C X_{A}-D X_{B}\right] X_{A}=\frac{B D-2 A C}{\left(4 A^{2}-B^{2}\right)^{2}} \frac{2 \pi}{\sqrt{4 A^{2}-B^{2}}} \exp \left\{-\frac{B C D-A\left(C^{2}+D^{2}\right)}{4 A^{2}-B^{2}}\right\} \\
& \int_{-\infty}^{\infty} d X_{A} \int_{-\infty}^{\infty} d X_{B} \exp \left[-A\left(X_{A}^{2}+X_{B}^{2}\right)-B X_{A} X_{B}-C X_{A}-D X_{B}\right]=\frac{2 \pi}{\sqrt{4 A^{2}-B^{2}}} \exp \left\{-\frac{B C D-A\left(C^{2}+D^{2}\right)}{4 A^{2}-B^{2}}\right\}
\end{aligned}
$$

${ }^{1}$ B. J. Berne, G. Ciccotti, and D. F. Coker, Classical and Quantum Dynamics in Condensed Phase Simulations (World Scientific, London, 1998).

${ }^{2}$ N. Makri, Annu. Rev. Phys. Chem. 50, 167 (1999).

${ }^{3}$ P. Jungwirth and R. B. Gerber, Chem. Rev. 99, 1583 (1999).

${ }^{4}$ T. Yamamoto, J. Chem. Phys. 33, 281 (1960).

${ }^{5}$ W. H. Miller, J. Chem. Phys. 61, 1823 (1974).

${ }^{6}$ W. H. Miller, S. D. Schwartz, and J. W. Tromp, J. Chem. Phys. 79, 4889 (1983).

${ }^{7}$ P. Hänggi, P. Talkner, and M. Borkovec, Rev. Mod. Phys. 62, 251 (1990).

${ }^{8}$ G. A. Voth, D. Chandler, and W. H. Miller, J. Chem. Phys. 91, 7749 (1989).

${ }^{9}$ G. A. Voth, Adv. Chem. Phys. 93, 135 (1996).

${ }^{10}$ M. Topaler and N. Makri, J. Chem. Phys. 101, 7500 (1994).

${ }^{11}$ N. Makri and K. Thompson, Chem. Phys. Lett. 291, 101 (1998).

${ }^{12}$ K. Yamashita and W. H. Miller, J. Chem. Phys. 82, 5475 (1985).

${ }^{13}$ E. Rabani, G. Krilov, and B. J. Berne, J. Chem. Phys. 112, 2605 (2000).

${ }^{14}$ E. Sim, G. Krilov, and B. Berne, J. Phys. Chem. A 105, 2824 (2001).

${ }^{15}$ E. Geva, Q. Shi, and G. A. Voth, J. Chem. Phys. 115, 9209 (2001).

${ }^{16}$ Q. Shi and E. Geva, J. Chem. Phys. 116, 3223 (2002).

${ }^{17}$ I. Navrotskaya, Q. Shi, and E. Geva, Isr. J. Chem. 42, 225 (2002).

${ }^{18}$ D. W. Oxtoby, Adv. Chem. Phys. 47, 487 (1981).

${ }^{19}$ D. W. Oxtoby, Annu. Rev. Phys. Chem. 32, 77 (1981).

${ }^{20}$ D. W. Oxtoby, J. Phys. Chem. 87, 3028 (1983).

${ }^{21}$ J. Chesnoy and G. M. Gale, Ann. Phys. (Paris) 9, 893 (1984).

${ }^{22}$ J. Chesnoy and G. M. Gale, Adv. Chem. Phys. 70, 297 (1988).

${ }^{23}$ C. B. Harris, D. E. Smith, and D. J. Russell, Chem. Rev. 90, 481 (1990).

${ }^{24}$ D. W. Miller and S. A. Adelman, Int. Rev. Phys. Chem. 13, 359 (1994).

${ }^{25}$ R. M. Stratt and M. Maroncelli, J. Phys. Chem. 100, 12981 (1996).

${ }^{26}$ T. Elsaesser and W. Kaiser, Annu. Rev. Phys. Chem. 42, 83 (1991).
${ }^{27}$ J. C. Owrutsky, D. Raftery, and R. M. Hochstrasser, Annu. Rev. Phys. Chem. 45, 519 (1994).

${ }^{28}$ S. A. Egorov and J. L. Skinner, J. Chem. Phys. 105, 7047 (1996).

${ }^{29}$ S. A. Egorov and J. L. Skinner, J. Chem. Phys. 106, 1034 (1997).

${ }^{30}$ S. A. Egorov and B. J. Berne, J. Chem. Phys. 107, 6050 (1997).

${ }^{31}$ S. A. Egorov, K. F. Everitt, and J. L. Skinner, J. Phys. Chem. A 103, 9494 (1999).

${ }^{32}$ S. A. Egorov and J. L. Skinner, J. Chem. Phys. 112, 275 (2000).

${ }^{33}$ K. F. Everitt, S. A. Egorov, and J. L. Skinner, Chem. Phys. 235, 115 (1998).

${ }^{34}$ K. F. Everitt and J. L. Skinner, J. Chem. Phys. 110, 4467 (1999).

${ }^{35}$ K. F. Everitt, J. L. Skinner, and B. M. Ladanyi, J. Chem. Phys. 116, 179 (2002).

${ }^{36}$ E. Rabani and D. R. Reichman, J. Phys. Chem. B 105, 6550 (2001).

${ }^{37}$ J. Poulsen, S. R. Keiding, and P. J. Rossky, Chem. Phys. Lett. 336, 488 (2001).

${ }^{38}$ J. Poulsen and P. J. Rossky, J. Chem. Phys. 115, 8014 (2001).

${ }^{39}$ J. Poulsen and P. J. Rossky, J. Chem. Phys. 115, 8024 (2001).

${ }^{40}$ H. Gai and G. A. Voth, J. Chem. Phys. 99, 740 (1993).

${ }^{41}$ H. Gai and G. A. Voth, J. Chem. Phys. 101, 1734 (1994).

${ }^{42}$ S. Jang, Y. Pak, and G. A. Voth, J. Phys. Chem. A 103, 10289 (1999).

${ }^{43}$ Q. Shi and E. Geva, J. Chem. Phys. 118, 7562 (2003).

${ }^{44}$ E. T. J. Nibbering, D. A. Wiersma, and K. Duppen, Phys. Rev. Lett. 66, 2464 (1991).

${ }^{45}$ E. T. J. Nibbering, D. A. Wiersma, and K. Duppen, Chem. Phys. 183, 167 (1994).

${ }^{46}$ M. S. Pshenichnikov, K. Duppen, and D. A. Wiersma, Phys. Rev. Lett. 74, 674 (1995).

${ }^{47}$ W. P. de Boeij, M. S. Pshenichnikov, and D. A. Wiersma, Chem. Phys. Lett. 238, 1 (1995).

${ }^{48}$ P. Vöhringer et al., J. Chem. Phys. 102, 4027 (1995). 
${ }^{49}$ T.-S. Yang, P. Vöhringer, D. C. Arnett, and N. F. Scherer, J. Chem. Phys. 103, 8346 (1995).

${ }^{50}$ R. Zhang, T.-S. Yang, and A. B. Myers, Chem. Phys. Lett. 211, 541 (1993).

${ }^{51}$ T.-S. Yang, R. Zhang, and A. B. Myers, J. Chem. Phys. 100, 8573 (1994).

${ }^{52}$ P. Cong, Y. J. Yan, H. P. Deuel, and J. D. Simon, J. Chem. Phys. 100, 7855 (1994).

${ }^{53}$ T. Joo, Y. Jia, and G. R. Fleming, J. Chem. Phys. 102, 4063 (1995).

${ }^{54}$ T. Joo et al., J. Chem. Phys. 104, 6089 (1996).

${ }^{55}$ M. L. Horng, J. A. Gardecki, A. Papazyan, and M. Maroncelli, J. Phys. Chem. 99, 17311 (1995).

${ }^{56}$ L. Reynolds et al., J. Phys. Chem. 100, 10337 (1996).

${ }^{57}$ S. Mukamel, Principles of Nonlinear Optical Spectroscopy (Oxford, New York, 1995).

${ }^{58}$ P. C. Becker et al., Phys. Rev. Lett. 63, 505 (1989).

${ }^{59}$ W. P. de Boeij, M. S. Pshenichnikov, and D. A. Wiersma, Annu. Rev. Phys. Chem. 49, 99 (1998).

${ }^{60}$ C. J. Bardeen, G. Cerrulo, and C. V. Shank, Chem. Phys. Lett. 280, 127 (1997).

${ }^{61}$ M. Cho and G. R. Fleming, J. Chem. Phys. 98, 2848 (1993).

${ }^{62}$ J. D. Hybl, A. W. Albrecht, S. M. Gallagher-Faeder, and D. M. Jonas, Chem. Phys. Lett. 297, 307 (1998).

${ }^{63}$ D. Zimdars, S. S. Francis, F. C. Ferrante, and M. D. Fayer, J. Chem. Phys. 106, 7498 (1997).

${ }^{64}$ R. F. Loring and S. Mukamel, Chem. Phys. Lett. 114, 426 (1985).

${ }^{65}$ A. M. Walsh and R. F. Loring, Chem. Phys. Lett. 186, 77 (1991).

${ }^{66}$ N. E. Shemetulskis and R. F. Loring, J. Chem. Phys. 97, 1217 (1992).

${ }^{67}$ Y. J. Yan and S. Mukamel, J. Chem. Phys. 94, 179 (1991).

${ }^{68}$ L. E. Fried, N. Bernstein, and S. Mukamel, Phys. Rev. Lett. 68, 1842 (1992).

${ }^{69}$ S. J. Rosenthal et al., J. Mol. Liq. 60, 25 (1994).

${ }^{70}$ M. F. Emde, A. Baltuska, A. Kummrow, and M. S. Pshenichnikov, Phys. Rev. Lett. 80, 4645 (1998).

${ }^{71}$ M. Cho et al., J. Phys. Chem. 100, 11944 (1996).

${ }^{72}$ S. A. Passino, Y. Nagasawa, and G. R. Fleming, J. Chem. Phys. 107, 6094 (1997).

${ }^{73}$ S. A. Passino, Y. Nagasawa, T. Joo, and G. R. Fleming, J. Phys. Chem. A 101, 725 (1997).

${ }^{74}$ M. J. Lang, X. J. Jordanides, X. Song, and G. R. Fleming, J. Chem. Phys. 110, 5884 (1999).

${ }^{75}$ D. S. Larsen, K. Ohta, and G. R. Fleming, J. Chem. Phys. 111, 8970 (1999).

${ }^{76}$ K. F. Everitt, E. Geva, and J. L. Skinner, J. Chem. Phys. 114, 1326 (2001).

${ }^{77}$ K. F. Everitt and J. L. Skinner, Chem. Phys. 266, 197 (2001).

${ }^{78}$ G. R. Fleming and M. Cho, Annu. Rev. Phys. Chem. 47, 109 (1996).

${ }^{79}$ S. A. Egorov, M. D. Stephens, and J. L. Skinner, J. Chem. Phys. 107, 10485 (1997).

${ }^{80}$ R. Kosloff, J. Phys. Chem. 92, 2087 (1988).

${ }^{81}$ C. Leforestier et al., J. Comput. Phys. 94, 59 (1991).

${ }^{82} \mathrm{R}$. Kosloff, in Numerical Grid Methods and Their Application to Schrödinger's Equation, edited by C. Cerjan (Kluwer Academic, The Netherlands, 1993), p. 175.

${ }^{83}$ R. Kosloff, Annu. Rev. Phys. Chem. 45, 145 (1994).

${ }^{84} \mathrm{R}$. Kosloff, in Dynamics of Molecules and Chemical Reactions, edited by D. T. Haar (Dekker, New York, 1996), p. 185.

${ }^{85} \mathrm{~K}$. Blum, Density Matrix Theory and Applications (Plenum, New York, 1996).

${ }^{86}$ S. Nakajima, Prog. Theor. Phys. 20, 948 (1958).

${ }^{87}$ R. Zwanzig, Lect. Theor. Phys. 3, 106 (1960).

${ }^{88}$ R. Zwanzig, J. Chem. Phys. 33, 1338 (1960).

${ }^{89}$ R. Zwanzig, Physica 30, 1109 (1964).

${ }^{90}$ I. Prigogine and P. Resibois, Physica 27, 629 (1961).

${ }^{91}$ F. Haake, Springer Tracts Mod. Phys. 66, 98 (1973).

${ }^{92} \mathrm{H}$. Grabert, Projection Operator Techniques in Nonequilibrium Statistical Mechanics (Springer, Berlin, 1982).

${ }^{93} \mathrm{R}$. Alicki and K. Lendi, Quantum Dynamical Semigroups and Applications (Springer, Berlin, 1987).

${ }^{94} \mathrm{~V}$. May and O. Kühn, Charge and Energy Transfer Dynamics in Molecular Systems (Wiley, Berlin, 2000).

${ }^{95}$ R. K. Wangsness and F. Bloch, Phys. Rev. 89, 728 (1953).

${ }^{96}$ A. G. Redfield, IBM Syst. J. 1, 19 (1957).

${ }^{97}$ B. Yoon, J. M. Deutch, and J. H. Freed, J. Chem. Phys. 62, 4687 (1975).

${ }^{98}$ I. Oppenheim, K. E. Shuler, and G. H. Weiss, Stochastic Processes in
Chemical Physics: The Master Equation (MIT Press, Cambridge, MA, 1977).

${ }^{99}$ S. Mukamel, I. Oppenheim, and J. Ross, Phys. Rev. A 17, 1988 (1978).

${ }^{100}$ V. Romero-Rochin and I. Oppenheim, Physica A 155, 52 (1989).

${ }^{101}$ V. Romero-Rochin, A. Orsky, and I. Oppenheim, Physica A 156, 244 (1989).

${ }^{102}$ N. G. van Kampen, Stochastic Processes in Physics and Chemistry (North-Holland, Amsterdam, 1981).

${ }^{103}$ R. Kubo, M. Toda, and N. Hashitsume, Statistical Physics II: Nonequilibrium Statistical Mechanics (Springer, New York, 1985).

${ }^{104}$ B. B. Laird, J. Budimir, and J. L. Skinner, J. Chem. Phys. 94, 4391 (1991).

${ }^{105}$ W. T. Pollard and R. A. Friesner, J. Chem. Phys. 100, 5054 (1994).

${ }^{106}$ W. T. Pollard, A. K. Felts, and R. A. Friesner, Adv. Chem. Phys. XCIII, 77 (1996).

${ }^{107}$ E. Geva, R. Kosloff, and J. L. Skinner, J. Chem. Phys. 102, 8541 (1995).

${ }^{108}$ E. Geva and R. Kosloff, J. Chem. Phys. 104, 7681 (1996).

${ }^{109}$ D. Kohen, C. C. Marston, and D. J. Tannor, J. Chem. Phys. 107, 5236 (1997).

${ }^{110}$ J. Cao, J. Chem. Phys. 107, 3204 (1997).

${ }^{111}$ Y. J. Yan, Phys. Rev. A 58, 2721 (1998).

${ }^{112}$ M. Berman, R. Kosloff, and H. Tal-Ezer, J. Phys. A 25, 1283 (1992).

${ }^{113}$ G. Ashkenazi et al., Adv. Chem. Phys. 100, 229 (1997).

${ }^{114}$ G. Ashkenazi, R. Kosloff, and M. A. Ratner, J. Am. Chem. Soc. 121, 3386 (1999).

${ }^{115}$ R. Kosloff, M. A. Ratner, and W. B. Davis, J. Chem. Phys. 106, 7036 (1997).

${ }^{116}$ B. B. Laird and J. L. Skinner, J. Chem. Phys. 94, 4405 (1991).

${ }^{117}$ D. R. Reichman and R. J. Silbey, J. Chem. Phys. 104, 1506 (1996).

${ }^{118}$ D. R. Reichman, F. L. H. B. Brown, and P. Neu, Phys. Rev. E 55, 2328 (1997).

${ }^{119}$ S. Jang, J. Cao, and R. J. Silbey, J. Chem. Phys. 116, 2705 (2002).

${ }^{120}$ A. Suárez and R. Silbey, J. Chem. Phys. 94, 4809 (1991).

${ }^{121} \mathrm{D}$. Li and G. A. Voth, J. Phys. Chem. 95, 10425 (1991).

${ }^{122}$ R. Silbey and R. A. Harris, J. Phys. Chem. 93, 7062 (1989).

${ }^{123}$ M. Sparpaglione and S. Mukamel, J. Chem. Phys. 88, 3263 (1988).

${ }^{124}$ A. A. Golosov and D. R. Reichman, J. Chem. Phys. 115, 9848 (2001).

${ }^{125}$ A. A. Golosov and D. R. Reichman, J. Chem. Phys. 115, 9862 (2001).

${ }^{126}$ R. P. Feynman and A. R. Hibbs, Quantum Mechanics and Path Integrals (McGraw-Hill, New York, 1965).

${ }^{127}$ L. S. Schulman, Techniques and Applications of Path Integration (Wiley, New York, 1981).

${ }^{128}$ H. Kleinert, Path Integrals in Quantum Mechanics, Statistics and Polymer Physics (World Scientific, NJ, 1995).

${ }^{129}$ R. P. Feynman and F. L. Vernon Jr., Ann. Phys. (N.Y.) 24, 118 (1963).

${ }^{130}$ P. G. Wolynes, Phys. Rev. Lett. 47, 968 (1981).

${ }^{131}$ A. O. Caldeira and A. J. Leggett, Ann. Phys. (N.Y.) 149, 374 (1983).

${ }^{132}$ R. D. Coalson, J. Chem. Phys. 86, 995 (1987).

${ }^{133}$ C. Mak and D. Chandler, Phys. Rev. A 44, 2352 (1991).

${ }^{134}$ M. Topaler and N. Makri, J. Chem. Phys. 97, 9001 (1992).

${ }^{135}$ M. Topaler and N. Makri, Chem. Phys. Lett. 210, 285 (1993).

${ }^{136}$ M. Topaler and N. Makri, Chem. Phys. Lett. 210, 448 (1993).

${ }^{137}$ M. Topaler and N. Makri, J. Phys. Chem. 100, 4430 (1996).

${ }^{138}$ D. E. Makarov and N. Makri, Chem. Phys. Lett. 221, 482 (1994).

${ }^{139}$ N. Makri and D. Makarov, J. Chem. Phys. 102, 4600 (1995).

${ }^{140}$ N. Makri and D. Makarov, J. Chem. Phys. 102, 4611 (1995).

${ }^{141}$ N. Makri, J. Math. Phys. 36, 2430 (1995).

${ }^{142}$ N. Makri, E. Sim, D. E. Makarov, and M. Topaler, Proc. Natl. Acad. Sci. U.S.A. 93, 3926 (1996).

${ }^{143}$ E. Sim and N. Makri, Comput. Phys. Commun. 99, 335 (1997).

${ }^{144}$ E. Sim and N. Makri, J. Phys. Chem. B 101, 5446 (1997).

${ }^{145}$ N. Makri, J. Phys. Chem. A 102, 4414 (1998).

${ }^{146}$ J. S. Shao and N. Makri, J. Chem. Phys. 116, 507 (2002),

${ }^{147}$ A. A. Golosov, R. A. Friesner, and P. Pechukas, J. Chem. Phys. 110, 138 (1999).

${ }^{148}$ A. A. Golosov, R. A. Friesner, and P. Pechukas, J. Chem. Phys. 112, 2095 (2000).

${ }^{149}$ K. Thompson and N. Makri, J. Chem. Phys. 13, 1343 (1999).

${ }^{150}$ K. Thompson and N. Makri, Phys. Rev. E 59, R4729 (1999).

${ }^{151}$ G. D. Billing, Chem. Phys. Lett. 30, 391 (1975).

${ }^{152}$ G. D. Billing, J. Chem. Phys. 99, 5849 (1993).

${ }^{153}$ J. C. Tully and R. K. Preston, J. Chem. Phys. 55, 562 (1971)

${ }^{154}$ J. C. Tully, J. Chem. Phys. 93, 1061 (1990).

${ }^{155}$ P. J. Kuntz, J. Chem. Phys. 95, 141 (1991). 
${ }^{156}$ A. I. Krylov et al., J. Chem. Phys. 104, 3651 (1996).

${ }^{157}$ E. Gallicchio and B. J. Berne, J. Chem. Phys. 105, 7064 (1996).

${ }^{158}$ E. Gallicchio, S. A. Egorov, and B. J. Berne, J. Chem. Phys. 109, 7745 (1998).

${ }^{159}$ S. A. Egorov, E. Gallicchio, and B. J. Berne, J. Chem. Phys. 107, 9312 (1997).

${ }^{160}$ G. Krilov and B. J. Berne, J. Chem. Phys. 111, 9147 (1999).

${ }^{161}$ E. Rabani, D. R. Reichman, G. Krylov, and B. J. Berne, Proc. Natl. Acad. Sci. U.S.A. 99, 1129 (2002).

${ }^{162}$ A. A. Golosov, D. R. Reichman, and E. Rabani, J. Chem. Phys. 118, 457 (2003).

${ }^{163}$ J. Cao and G. A. Voth, J. Chem. Phys. 100, 5106 (1994).

${ }^{164}$ J. Cao and G. A. Voth, J. Chem. Phys. 101, 6157 (1994).

${ }^{165}$ J. Cao and G. A. Voth, J. Chem. Phys. 101, 6168 (1994).

${ }^{166}$ S. Jang and G. A. Voth, J. Chem. Phys. 111, 2357 (1999).

${ }^{167}$ S. Jang and G. A. Voth, J. Chem. Phys. 111, 2371 (1999).

${ }^{168}$ D. R. Reichman, P.-N. Roy, S. Jang, and G. A. Voth, J. Chem. Phys. 113, 919 (2000).

${ }^{169}$ A. Calhoun, M. Pavese, and G. A. Voth, Chem. Phys. Lett. 262, 415 (1996).

${ }^{170}$ U. W. Schmitt and G. A. Voth, J. Chem. Phys. 111, 9361 (1999).

${ }^{171}$ M. Pavese and G. A. Voth, Chem. Phys. Lett. 249, 231 (1996).

${ }^{172}$ K. Kinugawa, P. B. Moore, and M. L. Klein, J. Chem. Phys. 106, 1154 (1997).

${ }^{173}$ K. Kinugawa, P. B. Moore, and M. L. Klein, J. Chem. Phys. 109, 610 (1998).

${ }^{174}$ K. Kinugawa, Chem. Phys. Lett. 292, 454 (1998).

${ }^{175}$ M. Pavese, D. R. Bernard, and G. A. Voth, Chem. Phys. Lett. 300, 93 (1999).

${ }^{176}$ D. R. Reichman and E. Rabani, Phys. Rev. Lett. 87, 265702 (2001).

${ }^{177}$ E. Rabani and D. R. Reichman, J. Chem. Phys. 116, 6271 (2002).
${ }^{178}$ E. Rabani and D. R. Reichman, Phys. Rev. E 65, 036111 (2002).

${ }^{179}$ D. R. Reichman and E. Rabani, J. Chem. Phys. 116, 6279 (2002).

${ }^{180}$ H. Wang, X. Sun, and W. H. Miller, J. Chem. Phys. 108, 9726 (1998).

${ }^{181}$ E. Pollak and J. Liao, J. Chem. Phys. 108, 2733 (1998).

${ }^{182}$ W. H. Miller, Adv. Chem. Phys. 25, 69 (1974).

${ }^{183}$ W. H. Miller, J. Chem. Phys. 53, 3578 (1970).

${ }^{184}$ M. F. Herman and E. Kluk, Chem. Phys. 91, 27 (1984).

${ }^{185}$ E. J. Heller, J. Chem. Phys. 94, 2723 (1981).

${ }^{186}$ K. G. Kay, J. Chem. Phys. 100, 4377 (1994).

${ }^{187}$ M. Ovchinnikov and V. A. Apkarian, J. Chem. Phys. 105, 10312 (1996).

${ }^{188}$ M. Ovchinnikov and V. A. Apkarian, J. Chem. Phys. 108, 2277 (1998).

${ }^{189}$ X. Sun and W. H. Miller, J. Chem. Phys. 106, 916 (1997).

${ }^{190}$ W. H. Miller, Faraday Discuss. 110, 1 (1998).

${ }^{191}$ J. S. Shao and N. Makri, J. Phys. Chem. A 103, 7753 (1999).

${ }^{192}$ K. Thompson and N. Makri, Phys. Rev. E 59, R4729 (1999).

${ }^{193}$ O. Kühn and N. Makri, J. Phys. Chem. A 103, 9487 (1999).

${ }^{194}$ H. Wang, M. Thoss, and W. H. Miller, J. Chem. Phys. 112, 47 (2000).

${ }^{195}$ M. Ovchinnikov, V. A. Apkarian, and G. A. Voth, J. Chem. Phys. 184, 7130 (2001).

${ }^{196}$ W. H. Miller, J. Phys. Chem. A 105, 2942 (2001).

${ }^{197}$ N. Makri and W. H. Miller, J. Chem. Phys. 116, 9207 (2002).

${ }^{198}$ A. Bartana, R. Kosloff, and D. J. Tannor, J. Chem. Phys. 106, 1435 (1997).

${ }^{199}$ E. Geva, J. Chem. Phys. 116, 1629 (2001).

${ }^{200}$ M. Demirplak and S. A. Rice, J. Chem. Phys. 116, 8028 (2002).

${ }^{201}$ Y. Ohtsuki, K. Nakagami, W. Zhu, and H. Rabitz, Chem. Phys. 287, 197 (2003).

${ }^{202}$ S. A. Rice and M. Zhao, Optical Control of Molecular Dynamics (Wiley, New York, 2000)

${ }^{203}$ W. H. Press, B. P. Flannery, S. A. Teukolsky, and W. T. Vetterling, Numerical Recipes (Cambridge University Press, Cambridge, MA, 1986). 